\title{
CORRIGENDA: \\ LOW-DIMENSIONAL REPRESENTATIONS \\ OF QUASI-SIMPLE GROUPS
}

\author{
GERHARD HISS AND GUNTER MALLE
}

\begin{abstract}
This paper contains corrections to the tables of low-dimensional representations of quasi-simple groups published in the paper, 'Lowdimensional representations of quasi-simple groups', LMS Journal of Computation and Mathematics 4 (2001) 22-63.
\end{abstract}

In our paper 'Low-dimensional representations of quasi-simple groups', we determine all the absolutely irreducible representations of quasi-simple groups of dimension at most 250, excluding those of groups of Lie type in their defining characteristic.

Martin Liebeck has kindly pointed out to us three omissions in our tables: the 12- and 13dimensional representations of the group $\mathrm{L}_{3}(3)$, and the 248-dimensional representations of $\mathrm{L}_{4}(5)$ in characteristic 2 .

When checking our arguments and calculations we realized that in fact all the representations of $\mathrm{L}_{3}(3)$ were missing, as well as the representations of $\mathrm{L}_{4}(5)$ of dimension exceeding 247.

The absolutely irreducible representations of $\mathrm{L}_{3}(3)$ can be found in the modular Atlas [7]. This leads to the first part of Table 1 below.

Table 1: The missing representations

\begin{tabular}{rlrlc}
\hline \multicolumn{1}{c}{$d$} & $G$ & \multicolumn{1}{c}{$\ell$} & field & ind \\
\hline 11 & $\mathrm{~L}_{3}(3)$ & 13 & & + \\
12 & $\mathrm{~L}_{3}(3)$ & 0,2 & & + \\
13 & $\mathrm{~L}_{3}(3)$ & 0,13 & & + \\
16 & $\mathrm{~L}_{3}(3)$ & 0,2 & $d 13$ & $\circ$ \\
16 & $\mathrm{~L}_{3}(3)$ & 13 & & + \\
26 & $\mathrm{~L}_{3}(3)$ & 0,13 & $i 2$ & $\circ$ \\
26 & $\mathrm{~L}_{3}(3)$ & $\neq 3$ & & + \\
27 & $\mathrm{~L}_{3}(3)$ & 0 & & + \\
39 & $\mathrm{~L}_{3}(3)$ & 0,13 & & + \\
248 & $2 . \mathrm{L}_{4}(5)$ & $\neq 2,5$ & & + \\
248 & $\mathrm{~L}_{4}(5)$ & 2 & & + \\
\hline
\end{tabular}

The absolutely irreducible representations of $\mathrm{L}_{4}(5)$ of degree up to 247 were classified by Guralnick and Tiep [3], and are contained in the original table. From the proofs given by Tiep 
and Zalesskii [9, Section 3], for example, it can be seen that the only other representations of degree at most 250 must arise as composition factors of the two ordinary 248-dimensional representations, which are both defined over the rationals [8, Proposition 13.5.6]. Since these characters are parametrized by elements of 2-power order in the dual group, [5, Proposition 1] shows that they remain irreducible for all primes $\ell \neq 2,5$. This yields the second-last entry in Table 1.

To obtain the last entry of our table, note first that, by Broué-Michel [1], the two ordinary representations of degree 248 lie in the principal 2-block of $\mathrm{L}_{4}(5)$. Using the decomposition numbers in [6], we find that the principal 2-block of $\mathrm{GL}_{4}(5)$ has irreducible Brauer characters of degrees 1, 154, 496, 3224, and 11904. By Clifford theory, the restriction to $\mathrm{SL}_{4}(5)$ of each of these characters has 1,2, or 4 irreducible constituents of the same degree. By the Seitz-Zalesskii bound, the smallest non-trivial representation of $\mathrm{SL}_{4}(5)$ has degree at least 152. Hence the character of degree 496 splits into two characters of degree 248 which are the reductions modulo 2 of the two ordinary characters of this degree. Jon Thackray has kindly constructed these representations over the field with two elements and computed their Frobenius-Schur indicators.

The existence of this representation had already been shown in [2].

Finally, Jon Thackray has pointed out to us that the Frobenius-Schur indicator for the 132-dimensional representations of the Harada-Norton group $\mathrm{HN}$ is - , rather than + , as given in our earlier table (see [4, Table 3]). This indicator has been known to the Atlas people for a long time.

For the convenience of the reader, we present the complete, corrected list of absolutely irreducible respresentations of quasi-simple groups in Table 2 below.

Table 2: Absolutely irreducible representations of quasi-simple groups, corrected

\begin{tabular}{llrlc}
\hline$d$ & $G$ & $\ell$ & field & ind \\
\hline 3 & $3 . \mathfrak{A}_{6}$ & 0,2 & $z 3, b 5$ & $\circ$ \\
3 & $3 . \mathfrak{A}_{6}$ & 5 & $z 3$ & $\circ$ \\
3 & $3 . \mathfrak{A}_{7}$ & 5 & $z 3, b 7$ & $\circ$ \\
4 & $\mathfrak{A}_{6}$ & 2 & & - \\
4 & $2 . \mathfrak{A}_{6}$ & 0,5 & & - \\
4 & $\mathfrak{A}_{7}$ & 2 & $b 7$ & $\circ$ \\
4 & $2 . \mathfrak{A}_{7}$ & 7 & & - \\
4 & $2 . \mathfrak{A}_{7}$ & 2,7 & $b 7$ & $\circ$ \\
4 & $42 . \mathrm{L}_{3}(4)$ & 3 & $i 1, r 7$ & $\circ$ \\
4 & $2 . \mathrm{U}_{4}(2)$ & 0,5 & $z 3$ & $\circ$ \\
5 & $\mathfrak{A}_{6}$ & 0,5 & & + \\
5 & $\mathfrak{A}_{7}$ & 7 & & $\circ$ \\
5 & $\mathrm{U}_{4}(2)$ & 0,5 & $z 3$ & $\circ$ \\
5 & $\mathrm{M}_{11}$ & 3 & $i 2, b 11$ & $\circ$ \\
6 & $3 . \mathfrak{A}_{6}$ & 0,5 & $z 3$ & $\circ$ \\
6 & $6 . \mathfrak{A}_{6}$ & 0,5 & $z 3, r 2$ & + \\
\hline
\end{tabular}


Corrigenda: Low-dimensional representations of quasi-simple groups

Table 2: Absolutely irreducible representations of quasi-simple groups, corrected

Continued from the previous page

\begin{tabular}{|c|c|c|c|c|}
\hline$d$ & $G$ & $\ell$ & field & ind \\
\hline 6 & $\mathfrak{A}_{7}$ & $\neq 7$ & & + \\
\hline 6 & $2 . \mathfrak{A}_{7}$ & 3 & $r 2$ & - \\
\hline 6 & $3 . \mathfrak{A}_{7}$ & $\neq 3$ & $z 3$ & ० \\
\hline 6 & $6 . \mathfrak{A}_{7}$ & $\neq 2,3$ & $z 3, r 2$ & $\circ$ \\
\hline 6 & 2. $\mathrm{L}_{3}(4)$ & 3 & & + \\
\hline 6 & $6 . \mathrm{L}_{3}(4)$ & $\neq 2,3$ & $z 3$ & ○ \\
\hline 6 & $\mathrm{U}_{3}(3)$ & $\neq 3$ & & - \\
\hline 6 & $\mathrm{U}_{4}(2)$ & 0,5 & & + \\
\hline 6 & $3_{1} \cdot \mathrm{U}_{4}(3)$ & 2 & $z 3$ & $\circ$ \\
\hline 6 & $6_{1} \cdot U_{4}(3)$ & $\neq 2,3$ & $z 3$ & $\circ$ \\
\hline 6 & $2 . \mathrm{M}_{12}$ & 3 & $i 2, i 5, b 11$ & $\circ$ \\
\hline 6 & $3 . \mathrm{M}_{22}$ & 2 & $z 3, b 11$ & ० \\
\hline 6 & $\mathrm{~J}_{2}$ & 2 & $b 5$ & - \\
\hline 6 & $2 . \mathrm{J}_{2}$ & 5 & & - \\
\hline 6 & $2 . \mathrm{J}_{2}$ & $\neq 2,5$ & $b 5$ & - \\
\hline 7 & $\mathfrak{A}_{8}$ & $\neq 2$ & & + \\
\hline 7 & $\mathfrak{A}_{9}$ & 3 & & + \\
\hline 7 & $\mathrm{U}_{3}(3)$ & 0,7 & & + \\
\hline 7 & $\mathrm{U}_{3}(3)$ & 0,7 & $i 1$ & $\circ$ \\
\hline 7 & $\mathrm{~S}_{6}(2)$ & $\neq 2$ & & + \\
\hline 7 & $\mathrm{~J}_{1}$ & 11 & $b 5, c 19$ & + \\
\hline 8 & $\mathfrak{A}_{6}$ & 0,2 & $b 5$ & + \\
\hline 8 & $\mathfrak{A}_{6}$ & 5 & & + \\
\hline 8 & $2 . \mathfrak{A}_{6}$ & 0 & $b 5$ & - \\
\hline 8 & $\mathfrak{A}_{7}$ & 5 & & + \\
\hline 8 & $2 . \mathfrak{A}_{8}$ & $\neq 2$ & & + \\
\hline 8 & $\mathfrak{A}_{9}$ & $\neq 3$ & & + \\
\hline 8 & 2.: $\mathfrak{A}_{9}$ & $\neq 2$ & & + \\
\hline 8 & $\mathfrak{A}_{10}$ & 2 & & - \\
\hline 8 & $\mathfrak{A}_{10}$ & 5 & & + \\
\hline 8 & $2 . \mathfrak{A}_{10}$ & 5 & $r 6, r 21$ & + \\
\hline 8 & $4_{1} \cdot \mathrm{L}_{3}(4)$ & 5 & $i 1$ & ○ \\
\hline 8 & $4_{1} \cdot \mathrm{L}_{3}(4)$ & $\neq 2,5$ & $i 1, b 5$ & ○ \\
\hline 8 & $2 . \mathrm{S}_{6}(2)$ & $\neq 2$ & & + \\
\hline 8 & $2 . \mathrm{O}_{8}^{+}(2)$ & $\neq 2$ & & + \\
\hline 8 & 2.Sz(8) & 5 & $c 13$ & + \\
\hline 9 & $\mathfrak{A}_{6}$ & 0 & & + \\
\hline 9 & $3 . \mathfrak{A}_{6}$ & 0,2 & $z 3$ & ० \\
\hline 9 & $3 . \mathfrak{A}_{7}$ & 7 & $z 3$ & $\circ$ \\
\hline 9 & $\mathfrak{A}_{10}$ & $\neq 2,5$ & & + \\
\hline
\end{tabular}


Corrigenda: Low-dimensional representations of quasi-simple groups

Table 2: Absolutely irreducible representations of quasi-simple groups, corrected

Continued from the previous page

\begin{tabular}{|c|c|c|c|c|}
\hline$d$ & $G$ & $\ell$ & field & ind \\
\hline 9 & $\mathfrak{A}_{11}$ & 11 & & + \\
\hline 9 & $\mathrm{M}_{11}$ & 11 & & + \\
\hline 9 & $3 . \mathrm{J}_{3}$ & 2 & $z 3, b 17, b 19$ & $\circ$ \\
\hline 10 & $\mathfrak{A}_{6}$ & 0,5 & & + \\
\hline 10 & $2 . \mathfrak{A}_{6}$ & 0,5 & $r 2$ & - \\
\hline 10 & $\mathfrak{A}_{7}$ & 7 & & + \\
\hline 10 & $\mathfrak{A}_{7}$ & $\neq 2,7$ & $b 7$ & $\circ$ \\
\hline 10 & $\mathfrak{A}_{11}$ & $\neq 11$ & & + \\
\hline 10 & $\mathfrak{A}_{12}$ & 2,3 & & + \\
\hline 10 & 2. $\mathrm{L}_{3}(4)$ & 7 & & + \\
\hline 10 & 2. $\mathrm{L}_{3}(4)$ & $\neq 2,7$ & $b 7$ & $\circ$ \\
\hline 10 & $\mathrm{U}_{4}(2)$ & 0,5 & $z 3$ & $\circ$ \\
\hline 10 & $\mathrm{U}_{5}(2)$ & $\neq 2$ & & - \\
\hline 10 & $\mathrm{M}_{11}$ & $\neq 11$ & & + \\
\hline 10 & $\mathrm{M}_{11}$ & $\neq 2$ & $i 2$ & $\circ$ \\
\hline 10 & $\mathrm{M}_{12}$ & 2,3 & & + \\
\hline 10 & 2. $\mathrm{M}_{12}$ & $\neq 2$ & $i 2$ & ○ \\
\hline 10 & $\mathrm{M}_{22}$ & 2 & $b 7$ & $\circ$ \\
\hline 10 & 2. $\mathrm{M}_{22}$ & 7 & & + \\
\hline 10 & 2. $\mathrm{M}_{22}$ & $\neq 2,7$ & $b 7$ & $\circ$ \\
\hline 11 & $\mathfrak{A}_{12}$ & $\neq 2,3$ & & + \\
\hline 11 & $\mathfrak{A}_{13}$ & 13 & & + \\
\hline 11 & $\mathrm{~L}_{3}(3)$ & 13 & & + \\
\hline 11 & $\mathrm{U}_{5}(2)$ & $\neq 2,3$ & $z 3$ & $\circ$ \\
\hline 11 & $\mathrm{M}_{11}$ & $\neq 2,3$ & & + \\
\hline 11 & $\mathrm{M}_{12}$ & $\neq 2,3$ & & + \\
\hline 11 & $\mathrm{M}_{23}$ & 2 & $b 7, i 15, b 23$ & $\circ$ \\
\hline 11 & $\mathrm{M}_{24}$ & 2 & $b 7, i 15, b 23$ & $\circ$ \\
\hline 12 & $6 . \mathfrak{A}_{6}$ & 0 & $z 3, b 5$ & $\circ$ \\
\hline 12 & $6 . \mathfrak{A}_{7}$ & 5 & $z 3, b 7$ & $\circ$ \\
\hline 12 & $\mathfrak{A}_{13}$ & $\neq 13$ & & + \\
\hline 12 & $\mathrm{~L}_{3}(3)$ & 0,2 & & + \\
\hline 12 & $12_{2} \cdot \mathrm{L}_{3}(4)$ & 7 & $z 12, b 5$ & $\circ$ \\
\hline 12 & $\mathrm{U}_{3}(4)$ & $\neq 2$ & & - \\
\hline 12 & $\mathrm{~S}_{4}(5)$ & 2 & $b 5$ & - \\
\hline 12 & $2 . \mathrm{S}_{4}(5)$ & $\neq 2,5$ & $b 5$ & - \\
\hline 12 & $2 . G_{2}(4)$ & $\neq 2$ & & - \\
\hline 12 & $2 . \mathrm{M}_{12}$ & $\neq 2,3$ & & + \\
\hline 12 & 2.Suz & 3 & & - \\
\hline 12 & 3.Suz & 2 & $z 3$ & $\circ$ \\
\hline
\end{tabular}


Corrigenda: Low-dimensional representations of quasi-simple groups

Table 2: Absolutely irreducible representations of quasi-simple groups, corrected

Continued from the previous page

\begin{tabular}{|c|c|c|c|c|}
\hline$d$ & $G$ & $\ell$ & field & ind \\
\hline 12 & 6.Suz & $\neq 2,3$ & $z 3$ & o \\
\hline 13 & $\mathfrak{A}_{7}$ & 3,5 & & + \\
\hline 13 & $\mathfrak{A}_{8}$ & 3,5 & & + \\
\hline 13 & $\mathrm{~L}_{3}(3)$ & 0,13 & & + \\
\hline 13 & $\mathrm{U}_{3}(4)$ & $\neq 2,5$ & $z 5$ & ○ \\
\hline 13 & $\mathrm{~S}_{4}(5)$ & $\neq 2,5$ & $b 5$ & + \\
\hline 13 & $\mathrm{~S}_{6}(3)$ & $\neq 3$ & $z 3$ & $\circ$ \\
\hline 13 & $\mathbf{J}_{2}$ & 3 & $b 5$ & + \\
\hline 14 & $\hat{\mathfrak{A}}_{7}$ & $\neq 3,5$ & & + \\
\hline 14 & $2 . \mathfrak{A}_{7}$ & $\neq 2,3$ & $r 2$ & - \\
\hline 14 & $\mathfrak{A}_{8}$ & 0,7 & & + \\
\hline 14 & $\mathrm{U}_{3}(3)$ & $\neq 3$ & & + \\
\hline 14 & $\mathrm{~S}_{6}(2)$ & 3 & & + \\
\hline 14 & $2 . S_{6}(3)$ & $\neq 2,3$ & $z 3$ & $\circ$ \\
\hline 14 & $\mathrm{Sz}(8)$ & $\neq 2$ & $i 1$ & $\circ$ \\
\hline 14 & $G_{2}(3)$ & $\neq 3$ & & + \\
\hline 14 & $\mathrm{~J}_{1}$ & 11 & $b 5, c 19$ & + \\
\hline 14 & $\mathrm{~J}_{2}$ & 5 & & + \\
\hline 14 & $\mathrm{~J}_{2}$ & $\neq 3,5$ & $b 5$ & + \\
\hline 14 & $2 . J_{2}$ & $\neq 2$ & & - \\
\hline 15 & $3 . \bar{A}_{6}$ & 0,5 & $z 3$ & ० \\
\hline 15 & $\mathfrak{A}_{7}$ & $\neq 2,7$ & & + \\
\hline 15 & $3 . \mathfrak{A}_{7}$ & $\neq 3$ & $z 3$ & ○ \\
\hline 15 & $\mathrm{~L}_{3}(4)$ & 3 & & + \\
\hline 15 & $3 . \mathrm{L}_{3}(4)$ & $\neq 2,3$ & $z 3$ & $\circ$ \\
\hline 15 & $\mathrm{U}_{4}(2)$ & 0,5 & & + \\
\hline 15 & $3_{1} \cdot \mathrm{U}_{4}(3)$ & $\neq 3$ & $z 3$ & ○ \\
\hline 15 & $\mathrm{~S}_{6}(2)$ & $\neq 2,3$ & & + \\
\hline 15 & $\mathrm{M}_{12}$ & 3 & $b 11$ & ० \\
\hline 15 & $3 . \mathrm{M}_{22}$ & 2 & $z 3, b 11$ & $\circ$ \\
\hline 16 & $2 . \mathfrak{A}_{7}$ & 7 & & - \\
\hline 16 & $2 . \mathfrak{A}_{8}$ & 7 & & - \\
\hline 16 & $\mathfrak{A}_{10}$ & 2 & & + \\
\hline 16 & $2 . \mathfrak{A}_{10}$ & $\neq 2,5$ & & + \\
\hline 16 & $\mathfrak{A}_{11}$ & 2 & $b 11$ & ० \\
\hline 16 & $2 . \mathfrak{A}_{11}$ & 11 & & + \\
\hline 16 & $2 . \mathfrak{A}_{11}$ & $\neq 2,11$ & $b 11$ & $\circ$ \\
\hline 16 & $\mathfrak{A}_{12}$ & 2 & $z 3$ & $\circ$ \\
\hline 16 & $2 . \mathfrak{A}_{12}$ & 3 & $i 2, i 5, r 7, b 11$ & $\circ$ \\
\hline 16 & $\mathrm{~L}_{3}(3)$ & 0,2 & $d 13$ & $\circ$ \\
\hline
\end{tabular}


Corrigenda: Low-dimensional representations of quasi-simple groups

Table 2: Absolutely irreducible representations of quasi-simple groups, corrected

Continued from the previous page

\begin{tabular}{|c|c|c|c|c|}
\hline$d$ & $G$ & $\ell$ & field & ind \\
\hline 16 & $\mathrm{~L}_{3}(3)$ & 13 & & + \\
\hline 16 & $4_{2} \cdot \mathrm{L}_{3}(4)$ & 3 & $i 1, r 7$ & $\circ$ \\
\hline 16 & 2.Sz(8) & 13 & $y 7$ & + \\
\hline 16 & $\mathrm{M}_{11}$ & 11 & & + \\
\hline 16 & $\mathrm{M}_{11}$ & $\neq 3,11$ & $b 11$ & ○ \\
\hline 16 & $\mathrm{M}_{12}$ & 11 & & + \\
\hline 16 & $\mathrm{M}_{12}$ & $\neq 3,11$ & $b 11$ & ० \\
\hline 16 & 4. $\mathrm{M}_{22}$ & 7 & $i 1, r 11$ & ० \\
\hline 18 & $3 . \mathfrak{A}_{7}$ & 5 & $z 3, b 7$ & o \\
\hline 18 & $\mathrm{~S}_{4}(4)$ & $\neq 2$ & & + \\
\hline 18 & $\mathrm{~J}_{3}$ & 3 & $b 5$ & + \\
\hline 18 & $3 . \mathrm{J}_{3}$ & 5 & $z 3$ & o \\
\hline 18 & $3 . \mathrm{J}_{3}$ & $\neq 3,5$ & $z 3, b 5$ & $\circ$ \\
\hline 19 & $\mathfrak{A}_{8}$ & 7 & & + \\
\hline 19 & $\mathfrak{A}_{9}$ & 7 & & + \\
\hline 19 & $\mathrm{~L}_{3}(4)$ & 3,7 & & + \\
\hline 20 & $\mathfrak{A}_{7}$ & 2 & & - \\
\hline 20 & $2 . \mathfrak{A}_{7}$ & $\neq 2,3$ & & - \\
\hline 20 & $\mathfrak{A}_{8}$ & 0,5 & & + \\
\hline 20 & $\mathfrak{A}_{9}$ & 2 & $i 15$ & $\circ$ \\
\hline 20 & $\mathrm{~L}_{3}(4)$ & 0,5 & & + \\
\hline 20 & $4_{2} \cdot \mathrm{L}_{3}(4)$ & $\neq 2,3$ & $i 1$ & o \\
\hline 20 & $\mathrm{U}_{3}(5)$ & $\neq 5$ & & - \\
\hline 20 & $\mathrm{U}_{4}(2)$ & 0,5 & & + \\
\hline 20 & 2. $\mathrm{U}_{4}(2)$ & 0,5 & & - \\
\hline 20 & 2. $\mathrm{U}_{4}(2)$ & 0,5 & $z 3$ & $\circ$ \\
\hline 20 & $\mathrm{U}_{4}(3)$ & 2 & & + \\
\hline 20 & 2. $\mathrm{U}_{4}(3)$ & $\neq 2,3$ & & - \\
\hline 20 & $4 . U_{4}(3)$ & $\neq 2,3$ & $i 1$ & ० \\
\hline 20 & $\mathrm{M}_{22}$ & 11 & & + \\
\hline 20 & $\mathrm{~J}_{1}$ & 2 & & + \\
\hline 20 & HS & 2 & & - \\
\hline 21 & $\mathfrak{A}_{7}$ & 0,7 & & + \\
\hline 21 & $3 . \mathfrak{A}_{7}$ & $\neq 2,3$ & $z 3$ & ० \\
\hline 21 & $\mathfrak{A}_{8}$ & 0,7 & $i 15$ & ० \\
\hline 21 & $\mathfrak{A}_{8}$ & $\neq 2$ & & + \\
\hline 21 & $\mathfrak{A}_{9}$ & 0,7 & $i 15$ & $\circ$ \\
\hline 21 & $\mathfrak{A}_{9}$ & 3,5 & & + \\
\hline 21 & $3 . \mathrm{L}_{3}(4)$ & $\neq 2,3$ & $z 3$ & ○ \\
\hline 21 & $\mathrm{U}_{3}(3)$ & 0,7 & & + \\
\hline
\end{tabular}


Corrigenda: Low-dimensional representations of quasi-simple groups

Table 2: Absolutely irreducible representations of quasi-simple groups, corrected

Continued from the previous page

\begin{tabular}{|c|c|c|c|c|}
\hline$d$ & $G$ & $\ell$ & field & ind \\
\hline 21 & $\mathrm{U}_{3}(3)$ & 0,7 & $i 1$ & o \\
\hline 21 & $\mathrm{U}_{3}(5)$ & $\neq 2,5$ & & + \\
\hline 21 & $3 . U_{3}(5)$ & $\neq 3,5$ & $z 3$ & ० \\
\hline 21 & $\mathrm{U}_{4}(3)$ & $\neq 2,3$ & & + \\
\hline 21 & $3_{1} \cdot \mathrm{U}_{4}(3)$ & $\neq 2,3$ & $z 3$ & $\circ$ \\
\hline 21 & $\mathrm{U}_{6}(2)$ & 3 & & + \\
\hline 21 & $3 . \mathrm{U}_{6}(2)$ & $\neq 2,3$ & $z 3$ & $\circ$ \\
\hline 21 & $\mathrm{~S}_{6}(2)$ & $\neq 2$ & & + \\
\hline 21 & $\mathrm{M}_{22}$ & $\neq 2,11$ & & + \\
\hline 21 & 3. $\mathrm{M}_{22}$ & $\neq 2,3$ & $z 3$ & $\circ$ \\
\hline 21 & $\mathrm{M}_{23}$ & 23 & & + \\
\hline 21 & $\mathrm{~J}_{2}$ & 5 & & + \\
\hline 21 & $\mathbf{J}_{2}$ & $\neq 2,5$ & $b 5$ & + \\
\hline 21 & HS & 5 & & + \\
\hline 21 & $\mathrm{McL}$ & 3,5 & & + \\
\hline 22 & $2 . \mathrm{L}_{3}(4)$ & 3 & $b 5$ & + \\
\hline 22 & $\mathrm{U}_{6}(2)$ & $\neq 2,3$ & & + \\
\hline 22 & $\mathrm{M}_{23}$ & $\neq 2,23$ & & + \\
\hline 22 & $\mathrm{M}_{24}$ & 3 & & + \\
\hline 22 & $\mathrm{~J}_{1}$ & 19 & $b 5$ & + \\
\hline 22 & HS & $\neq 2,5$ & & + \\
\hline 22 & $\mathrm{McL}$ & $\neq 3,5$ & & + \\
\hline 22 & $\mathrm{Co}_{3}$ & 2 & & - \\
\hline 22 & $\mathrm{Co}_{3}$ & 3 & & + \\
\hline 22 & $\mathrm{Co}_{2}$ & 2 & & + \\
\hline 23 & $\mathrm{U}_{4}(2)$ & 5 & & + \\
\hline 23 & $\mathrm{M}_{24}$ & $\neq 2,3$ & & + \\
\hline 23 & $\mathrm{Co}_{3}$ & $\neq 2,3$ & & + \\
\hline 23 & $\mathrm{Co}_{2}$ & $\neq 2$ & & + \\
\hline 24 & $3 . \mathfrak{A}_{7}$ & 0,2 & $z 3, b 7$ & $\circ$ \\
\hline 24 & $6 . \mathfrak{A}_{7}$ & 7 & $z 3$ & $\circ$ \\
\hline 24 & $6 . \mathfrak{A}_{7}$ & 0,5 & $z 3, b 7$ & $\circ$ \\
\hline 24 & $2 . \mathfrak{A}_{8}$ & $\neq 2,7$ & $b 7$ & $\circ$ \\
\hline 24 & $4_{1} \cdot \mathrm{L}_{3}(4)$ & 3 & $i 1, r 7$ & $\circ$ \\
\hline 24 & $12_{1} \cdot \mathrm{L}_{3}(4)$ & 7 & $z 12$ & ० \\
\hline 24 & $12_{1} \cdot \mathrm{L}_{3}(4)$ & 0,5 & $z 12, b 7$ & $\circ$ \\
\hline 24 & $\mathrm{U}_{4}(2)$ & 0 & & + \\
\hline 24 & $\mathrm{~S}_{4}(7)$ & 2 & $b 7$ & ○ \\
\hline 24 & $2 . \mathrm{S}_{4}(7)$ & $\neq 2,7$ & $b 7$ & $\circ$ \\
\hline 24 & 2.Sz(8) & 13 & $y 7$ & + \\
\hline
\end{tabular}


Corrigenda: Low-dimensional representations of quasi-simple groups

Table 2: Absolutely irreducible representations of quasi-simple groups, corrected

Continued from the previous page

\begin{tabular}{|c|c|c|c|c|}
\hline$d$ & $G$ & $\ell$ & field & ind \\
\hline 24 & $\mathrm{M}_{11}$ & 3 & & + \\
\hline 24 & 12. $\mathrm{M}_{22}$ & 11 & $z 24, b 7$ & $\circ$ \\
\hline 24 & $\mathrm{Co}_{1}$ & 2 & & + \\
\hline 24 & 2. $\mathrm{Co}_{1}$ & $\neq 2$ & & + \\
\hline 25 & $\mathrm{~S}_{4}(7)$ & $\neq 2,7$ & $b 7$ & o \\
\hline 25 & ${ }^{3} D_{4}(2)$ & 3 & & + \\
\hline 26 & $\mathfrak{A}_{9}$ & 2 & & + \\
\hline 26 & $\mathfrak{A}_{10}$ & 2 & & + \\
\hline 26 & $\mathrm{~L}_{3}(3)$ & 0,13 & $i 2$ & $\circ$ \\
\hline 26 & $\mathrm{~L}_{3}(3)$ & $\neq 3$ & & + \\
\hline 26 & $2 . \mathrm{L}_{3}(4)$ & 7 & & + \\
\hline 26 & $\mathrm{~L}_{4}(3)$ & $\neq 3$ & & + \\
\hline 26 & $\mathrm{U}_{3}(3)$ & 7 & & + \\
\hline 26 & $S_{6}(2)$ & 7 & & + \\
\hline 26 & ${ }^{3} D_{4}(2)$ & $\neq 2,3$ & & + \\
\hline 26 & ${ }^{2} F_{4}(2){ }^{\prime}$ & 2 & & + \\
\hline 26 & ${ }^{2} F_{4}(2){ }^{\prime}$ & $\neq 2$ & $i 2$ & $\circ$ \\
\hline 27 & $\mathfrak{A}_{9}$ & $\neq 2,7$ & & + \\
\hline 27 & $\mathrm{~L}_{3}(3)$ & 0 & & + \\
\hline 27 & $\mathrm{U}_{3}(3)$ & 0 & & + \\
\hline 27 & $S_{6}(2)$ & $\neq 2,7$ & & + \\
\hline 27 & $3 . \mathrm{O}_{7}(3)$ & $\neq 3$ & $z 3$ & $\circ$ \\
\hline 27 & $3 . G_{2}(3)$ & $\neq 3$ & $z 3$ & ○ \\
\hline 27 & ${ }^{2} F_{4}(2)^{\prime}$ & $\neq 2$ & $i 1$ & $\circ$ \\
\hline 27 & $\mathrm{~J}_{1}$ & 11 & $b 5, c 19$ & + \\
\hline 27 & 3. $\mathrm{Fi}_{22}$ & 2 & $z 3, b 11$ & $\circ$ \\
\hline 28 & $\mathfrak{A}_{8}$ & $\neq 2,5$ & & + \\
\hline 28 & $\mathfrak{A}_{9}$ & $\neq 2,3$ & & + \\
\hline 28 & $\mathfrak{A}_{10}$ & 5 & & + \\
\hline 28 & $2 . \mathrm{L}_{3}(4)$ & 5 & & + \\
\hline 28 & 2. $\mathrm{L}_{3}(4)$ & 0,7 & $b 5$ & + \\
\hline 28 & $4_{2} \cdot \mathrm{L}_{3}(4)$ & 5 & $i 1$ & ० \\
\hline 28 & $4_{2} \cdot \mathrm{L}_{3}(4)$ & $\neq 2,5$ & $i 1, b 5$ & $\circ$ \\
\hline 28 & $\mathrm{U}_{3}(3)$ & 0,7 & $i 1$ & o \\
\hline 28 & $\mathrm{U}_{3}(5)$ & $\neq 5$ & & + \\
\hline 28 & $\mathrm{O}_{8}^{+}(2)$ & $\neq 2$ & & + \\
\hline 28 & $2 . \mathrm{M}_{22}$ & 5 & $i 1, r 11$ & o \\
\hline 28 & 2.HS & 5 & $i 1, r 11$ & o \\
\hline 28 & $\mathrm{Ru}$ & 2 & & + \\
\hline 28 & 2. $\mathrm{Ru}$ & $\neq 2$ & $i 1$ & ० \\
\hline
\end{tabular}


Corrigenda: Low-dimensional representations of quasi-simple groups

Table 2: Absolutely irreducible representations of quasi-simple groups, corrected

Continued from the previous page

\begin{tabular}{|c|c|c|c|c|}
\hline$d$ & $G$ & $\ell$ & field & ind \\
\hline 29 & $\mathrm{~L}_{3}(5)$ & 31 & & + \\
\hline 29 & $\mathrm{~L}_{5}(2)$ & 31 & & + \\
\hline 29 & $\mathrm{M}_{12}$ & 11 & & + \\
\hline 30 & $\mathrm{~L}_{3}(5)$ & $\neq 5,31$ & & + \\
\hline 30 & $\mathrm{~L}_{5}(2)$ & $\neq 2,31$ & & + \\
\hline 30 & $\mathrm{U}_{4}(2)$ & 0,5 & & + \\
\hline 30 & $\mathrm{U}_{4}(2)$ & 0,5 & $z 3$ & o \\
\hline 31 & $\mathrm{~L}_{3}(5)$ & $\neq 2,5$ & & + \\
\hline 31 & $\mathrm{~L}_{3}(5)$ & $\neq 2,5$ & $i 1$ & $\circ$ \\
\hline 31 & $\mathrm{~J}_{1}$ & 7 & $c 19$ & + \\
\hline 32 & $2 . \mathfrak{A}_{8}$ & 5 & $z 3, b 7$ & ० \\
\hline 32 & $2 . \mathfrak{A}_{12}$ & $\neq 2,3$ & & - \\
\hline 32 & $\mathfrak{A}_{13}$ & 2 & $b 13$ & + \\
\hline 32 & $2 . \mathfrak{A}_{13}$ & 13 & & - \\
\hline 32 & $2 . \mathfrak{A}_{13}$ & $\neq 2,13$ & $b 13$ & - \\
\hline 32 & $2 . \mathfrak{A}_{14}$ & 7 & $r 3, r 6, r 10, b 5, b 13, b 33$ & - \\
\hline 32 & $\mathrm{U}_{3}(3)$ & 0,2 & $b 7$ & $\circ$ \\
\hline 32 & 2. $\mathrm{U}_{4}(2)$ & 5 & $z 3$ & $\circ$ \\
\hline 32 & 2. $\mathrm{M}_{12}$ & $\neq 2,3$ & & - \\
\hline 33 & $\mathrm{~S}_{4}(4)$ & 5 & & + \\
\hline 33 & $\mathrm{O}_{8}^{-}(2)$ & 7 & & + \\
\hline 34 & $\mathfrak{A}_{9}$ & 5 & & + \\
\hline 34 & $\mathfrak{A}_{10}$ & 3,5 & & + \\
\hline 34 & $\mathfrak{A}_{11}$ & 3 & & + \\
\hline 34 & $\mathrm{U}_{4}(3)$ & 2 & & - \\
\hline 34 & $\mathrm{~S}_{4}(4)$ & $\neq 2,5$ & & + \\
\hline 34 & $\mathrm{~S}_{6}(2)$ & 3 & & + \\
\hline 34 & $\mathrm{O}_{8}^{-}(2)$ & $\neq 2,7$ & & + \\
\hline 34 & $\mathrm{M}_{12}$ & 3 & & + \\
\hline 34 & $\mathrm{M}_{22}$ & 2 & & - \\
\hline 34 & $\mathrm{~J}_{1}$ & 19 & $b 5$ & + \\
\hline 35 & $\mathfrak{A}_{7}$ & $\neq 2,3$ & & + \\
\hline 35 & $\mathfrak{A}_{8}$ & $\neq 2$ & & + \\
\hline 35 & $\mathfrak{A}_{9}$ & $\neq 2$ & & + \\
\hline 35 & $\mathfrak{A}_{10}$ & $\neq 2,3$ & & + \\
\hline 35 & $\mathfrak{A}_{10}$ & 5 & $r 21$ & + \\
\hline 35 & $\mathrm{~L}_{3}(4)$ & $\neq 2,3$ & & + \\
\hline 35 & $\mathrm{U}_{4}(3)$ & $\neq 2,3$ & & + \\
\hline 35 & $\mathrm{~S}_{6}(2)$ & $\neq 2$ & & + \\
\hline 35 & $\mathrm{~S}_{8}(2)$ & $\neq 2$ & & + \\
\hline
\end{tabular}


Corrigenda: Low-dimensional representations of quasi-simple groups

Table 2: Absolutely irreducible representations of quasi-simple groups, corrected

Continued from the previous page

\begin{tabular}{|c|c|c|c|c|}
\hline$d$ & $G$ & $\ell$ & field & ind \\
\hline 35 & $\mathrm{O}_{8}^{+}(2)$ & $\neq 2$ & & + \\
\hline 35 & $\mathrm{Sz}(8)$ & 13 & & + \\
\hline 35 & $\mathrm{Sz}(8)$ & $\neq 2,13$ & $c 13$ & + \\
\hline 36 & $2 . \mathfrak{A}_{7}$ & 0,3 & & - \\
\hline 36 & $6 . \mathfrak{A}_{7}$ & 0 & $z 3$ & o \\
\hline 36 & $\mathfrak{A}_{10}$ & $\neq 2,5$ & & + \\
\hline 36 & $\mathfrak{A}_{11}$ & 11 & & + \\
\hline 36 & $2 . \mathrm{L}_{3}(4)$ & $\neq 2,7$ & & + \\
\hline 36 & $4_{2} \cdot \mathrm{L}_{3}(4)$ & $\neq 2$ & $i 1$ & ० \\
\hline 36 & $6 . \mathrm{L}_{3}(4)$ & $\neq 2,3$ & $z 3$ & ○ \\
\hline 36 & $12_{2} \cdot \mathrm{L}_{3}(4)$ & $\neq 2,3$ & $z 12$ & ○ \\
\hline 36 & 2. $\mathrm{U}_{4}(2)$ & 0 & $z 3$ & $\circ$ \\
\hline 36 & $3_{2} \cdot \mathrm{U}_{4}(3)$ & $\neq 3$ & $z 3$ & $\circ$ \\
\hline 36 & $12_{2} \cdot \mathrm{U}_{4}(3)$ & $\neq 2,3$ & $z 12$ & $\circ$ \\
\hline 36 & $6 . \mathrm{M}_{22}$ & 11 & $z 12$ & ○ \\
\hline 36 & $\mathbf{J}_{2}$ & $\neq 5$ & & + \\
\hline 36 & $2 . \mathrm{J}_{2}$ & 3 & $i 1$ & o \\
\hline 38 & $\mathrm{~L}_{4}(3)$ & 2,5 & & + \\
\hline 39 & $\mathrm{~L}_{3}(3)$ & 0,13 & & + \\
\hline 39 & $\mathrm{~L}_{4}(3)$ & 0,13 & & + \\
\hline 39 & $\mathrm{U}_{3}(4)$ & 5 & & + \\
\hline 39 & $\mathrm{U}_{3}(4)$ & $\neq 2,5$ & $b 5$ & + \\
\hline 40 & $2 . \mathrm{L}_{4}(3)$ & $\neq 2,3$ & & + \\
\hline 40 & $4_{1} \cdot \mathrm{L}_{3}(4)$ & 3 & $i 1$ & ० \\
\hline 40 & $\mathrm{U}_{4}(2)$ & 0,5 & $z 3$ & $\circ$ \\
\hline 40 & $\mathrm{~S}_{4}(5)$ & $\neq 5$ & & + \\
\hline 40 & $\mathrm{~S}_{4}(9)$ & 2 & & - \\
\hline 40 & $2 . \mathrm{S}_{4}(9)$ & $\neq 2,3$ & & - \\
\hline 40 & $2 . S_{6}(2)$ & 7 & & + \\
\hline 40 & $\mathrm{~S}_{8}(3)$ & 2 & $z 3$ & ० \\
\hline 40 & $2 . \mathrm{S}_{8}(3)$ & $\neq 2,3$ & $z 3$ & $\circ$ \\
\hline 40 & 2.Sz(8) & 7 & & + \\
\hline 40 & $2 . \mathrm{Sz}(8)$ & $\neq 2,7$ & $y 7$ & + \\
\hline 41 & $\mathfrak{A}_{9}$ & 3 & & + \\
\hline 41 & $\mathfrak{A}_{10}$ & 3 & & + \\
\hline 41 & $\mathrm{~S}_{4}(9)$ & $\neq 2,3$ & & + \\
\hline 41 & $\mathrm{~S}_{8}(3)$ & $\neq 2,3$ & $z 3$ & o \\
\hline 41 & $\mathrm{~J}_{2}$ & 5 & & + \\
\hline 42 & $\mathfrak{A}_{9}$ & 0,7 & & + \\
\hline 42 & $\mathfrak{A}_{10}$ & 0,7 & & + \\
\hline
\end{tabular}


Corrigenda: Low-dimensional representations of quasi-simple groups

Table 2: Absolutely irreducible representations of quasi-simple groups, corrected

Continued from the previous page

\begin{tabular}{|c|c|c|c|c|}
\hline$d$ & $G$ & $\ell$ & field & ind \\
\hline 42 & $6 . \mathrm{L}_{3}(4)$ & 0,7 & $z 3, b 5$ & o \\
\hline 42 & $\mathrm{U}_{3}(7)$ & $\neq 7$ & & - \\
\hline 42 & $\mathrm{U}_{7}(2)$ & $\neq 2$ & & - \\
\hline 43 & $\mathfrak{A}_{8}$ & 5 & & + \\
\hline 43 & $\mathfrak{A}_{11}$ & 5 & & + \\
\hline 43 & $\mathfrak{A}_{12}$ & 5 & & + \\
\hline 43 & $\mathrm{U}_{3}(7)$ & $\neq 2,7$ & & + \\
\hline 43 & $\mathrm{U}_{3}(7)$ & $\neq 2,7$ & $i 1$ & $\circ$ \\
\hline 43 & $\mathrm{U}_{3}(7)$ & $\neq 2,7$ & $z 8$ & $\circ$ \\
\hline 43 & $\mathrm{U}_{5}(2)$ & 5 & & + \\
\hline 43 & $\mathrm{U}_{7}(2)$ & $\neq 2,3$ & $z 3$ & ० \\
\hline 43 & $\mathrm{~J}_{1}$ & 19 & $b 5$ & + \\
\hline 44 & $\mathfrak{A}_{11}$ & $\neq 3,5$ & & + \\
\hline 44 & $\mathfrak{A}_{12}$ & 2 & & + \\
\hline 44 & $4_{2} \cdot \mathrm{L}_{3}(4)$ & 7 & $i 1$ & $\circ$ \\
\hline 44 & $\mathrm{U}_{5}(2)$ & $\neq 2,5$ & & + \\
\hline 44 & $\mathrm{M}_{11}$ & $\neq 3,5$ & & + \\
\hline 44 & $\mathrm{M}_{12}$ & 2 & & + \\
\hline 44 & 2. $\mathrm{M}_{12}$ & $\neq 2,5$ & $i 5$ & $\circ$ \\
\hline 44 & $\mathrm{M}_{23}$ & 2 & $b 7$ & $\circ$ \\
\hline 44 & $\mathrm{M}_{24}$ & 2 & $b 7$ & $\circ$ \\
\hline 45 & $\mathfrak{A}_{8}$ & 7 & & + \\
\hline 45 & $\mathfrak{A}_{8}$ & $\neq 2,7$ & $b 7$ & ○ \\
\hline 45 & $\mathfrak{A}_{11}$ & $\neq 2,11$ & & + \\
\hline 45 & $\mathfrak{A}_{12}$ & 3 & & + \\
\hline 45 & $\mathrm{~L}_{3}(4)$ & 7 & & + \\
\hline 45 & $\mathrm{~L}_{3}(4)$ & $\neq 2,7$ & $b 7$ & ○ \\
\hline 45 & $3 . \mathrm{L}_{3}(4)$ & 0,5 & $z 3, b 7$ & $\circ$ \\
\hline 45 & $\mathrm{U}_{4}(2)$ & 0,5 & $z 3$ & $\circ$ \\
\hline 45 & $3_{2} \cdot \mathrm{U}_{4}(3)$ & 7 & $z 3$ & $\circ$ \\
\hline 45 & $3_{2} \cdot \mathrm{U}_{4}(3)$ & $\neq 3,7$ & $z 3, b 7$ & $\circ$ \\
\hline 45 & $\mathrm{M}_{11}$ & $\neq 2,11$ & & + \\
\hline 45 & $\mathrm{M}_{12}$ & $\neq 2,11$ & & + \\
\hline 45 & $\mathrm{M}_{22}$ & 7 & & + \\
\hline 45 & $\mathrm{M}_{22}$ & $\neq 2,7$ & $b 7$ & ० \\
\hline 45 & 3. $\mathrm{M}_{22}$ & 7 & $z 3$ & $\circ$ \\
\hline 45 & 3. $\mathrm{M}_{22}$ & $\neq 3,7$ & $z 3, b 7$ & $\circ$ \\
\hline 45 & $\mathrm{M}_{23}$ & 7 & & + \\
\hline 45 & $\mathrm{M}_{23}$ & $\neq 2,7$ & $b 7$ & 0 \\
\hline 45 & $\mathrm{M}_{24}$ & 7 & & + \\
\hline
\end{tabular}


Corrigenda: Low-dimensional representations of quasi-simple groups

Table 2: Absolutely irreducible representations of quasi-simple groups, corrected

Continued from the previous page

\begin{tabular}{|c|c|c|c|c|}
\hline$d$ & $G$ & $\ell$ & field & ind \\
\hline 45 & $\mathrm{M}_{24}$ & $\neq 2,7$ & $b 7$ & $\circ$ \\
\hline 45 & $\mathrm{~J}_{1}$ & 7 & $c 19$ & + \\
\hline 45 & 3.McL & 5 & $z 3, b 7$ & ० \\
\hline 45 & $3 . \mathrm{O}^{\prime} \mathrm{N}$ & 7 & & o \\
\hline 47 & $\mathfrak{A}_{9}$ & 7 & & + \\
\hline 48 & $2 . \mathfrak{A}_{8}$ & $\neq 2$ & & - \\
\hline 48 & $\mathfrak{A}_{9}$ & 0,2 & & + \\
\hline 48 & 2. $\mathfrak{A}_{9}$ & 3 & & + \\
\hline 48 & 2.: $\mathfrak{A}_{9}$ & $\neq 2,3$ & $i 6$ & $\circ$ \\
\hline 48 & $\mathfrak{A}_{10}$ & 2 & & + \\
\hline 48 & $2 . \mathfrak{A}_{10}$ & 3 & & + \\
\hline 48 & $2 . \mathfrak{A}_{10}$ & $\neq 2,3$ & $i 6$ & ० \\
\hline 48 & $12_{1} \cdot \mathrm{L}_{3}(4)$ & 0,7 & $z 12, b 5$ & $\circ$ \\
\hline 48 & $12_{2} \cdot \mathrm{L}_{3}(4)$ & 0,7 & $z 12, b 5$ & $\circ$ \\
\hline 48 & $12_{2} \cdot \mathrm{L}_{3}(4)$ & 5 & $z 12$ & $\circ$ \\
\hline 48 & $3 . U_{3}(5)$ & $\neq 3,5$ & $z 3$ & $\circ$ \\
\hline 48 & $2 . S_{6}(2)$ & $\neq 2,7$ & & + \\
\hline 48 & $\mathrm{O}_{8}^{+}(2)$ & 3 & & + \\
\hline 48 & 2.Sz(8) & 5 & $c 13$ & + \\
\hline 48 & $12 . \mathrm{M}_{22}$ & 5 & $z 12, b 11$ & o \\
\hline 49 & $\mathrm{~S}_{4}(4)$ & 17 & & + \\
\hline 49 & $\mathrm{~S}_{6}(2)$ & 3 & & + \\
\hline 49 & $\mathrm{M}_{22}$ & 3 & $b 11$ & ० \\
\hline 49 & $\mathrm{~J}_{1}$ & 11 & $b 5, c 19$ & + \\
\hline 49 & HS & 3 & $i 5, b 11$ & o \\
\hline 50 & $\mathrm{~S}_{4}(4)$ & $\neq 2,17$ & & + \\
\hline 50 & $\mathrm{~S}_{8}(2)$ & 3 & & + \\
\hline 50 & $\mathrm{O}_{8}^{+}(2)$ & $\neq 2,3$ & & + \\
\hline 50 & $\mathrm{O}_{8}^{-}(2)$ & 3 & & + \\
\hline 50 & $2 . J_{2}$ & 3 & $b 5$ & - \\
\hline 50 & $2 . \mathrm{J}_{2}$ & $\neq 2,3$ & $i 1$ & ○ \\
\hline 50 & $\mathrm{He}$ & 7 & & + \\
\hline 51 & $\mathrm{U}_{4}(4)$ & 5 & & + \\
\hline 51 & $\mathrm{U}_{4}(4)$ & $\neq 2,5$ & $z 5$ & $\circ$ \\
\hline 51 & $\mathrm{~S}_{4}(4)$ & $\neq 2,5$ & $b 5$ & + \\
\hline 51 & $\mathrm{~S}_{8}(2)$ & $\neq 2,3$ & & + \\
\hline 51 & $\mathrm{O}_{8}^{-}(2)$ & $\neq 2,3$ & & + \\
\hline 51 & $\mathrm{He}$ & $\neq 7$ & $b 7$ & $\circ$ \\
\hline 52 & $\mathrm{~L}_{4}(3)$ & $\neq 2,3$ & & + \\
\hline 52 & $\mathrm{U}_{3}(4)$ & $\neq 2,5$ & $z 5$ & $\circ$ \\
\hline
\end{tabular}


Corrigenda: Low-dimensional representations of quasi-simple groups

Table 2: Absolutely irreducible representations of quasi-simple groups, corrected

Continued from the previous page

\begin{tabular}{|c|c|c|c|c|}
\hline$d$ & $G$ & $\ell$ & field & ind \\
\hline 52 & $\mathrm{U}_{4}(4)$ & $\neq 2,5$ & & + \\
\hline 52 & $2 . S_{4}(5)$ & $\neq 2,5$ & $b 5$ & - \\
\hline 52 & ${ }^{3} D_{4}(2)$ & $\neq 2$ & & + \\
\hline 52 & $2 . F_{4}(2)$ & $\neq 2$ & & + \\
\hline 53 & $\mathfrak{A}_{12}$ & 11 & & + \\
\hline 53 & $\mathfrak{A}_{13}$ & 11 & & + \\
\hline 53 & $\mathrm{M}_{12}$ & 11 & & + \\
\hline 54 & $\mathfrak{A}_{12}$ & $0,3,7$ & & + \\
\hline 54 & $6 . \mathrm{L}_{3}(4)$ & 7 & $z 3$ & o \\
\hline 54 & $\mathrm{M}_{12}$ & 0,3 & & + \\
\hline 54 & $\mathrm{M}_{22}$ & 7 & & + \\
\hline 54 & $6 . \mathrm{M}_{22}$ & 7 & $z 3$ & ० \\
\hline 55 & $\mathfrak{A}_{10}$ & 5 & & + \\
\hline 55 & $\mathfrak{A}_{11}$ & 5 & & + \\
\hline 55 & $\mathfrak{A}_{12}$ & $\neq 2,3$ & & + \\
\hline 55 & $\mathfrak{A}_{13}$ & 13 & & + \\
\hline 55 & $\mathrm{~L}_{3}(7)$ & 3,19 & & + \\
\hline 55 & $\mathrm{U}_{5}(2)$ & $\neq 2$ & & + \\
\hline 55 & $\mathrm{U}_{5}(2)$ & $\neq 2,3$ & $z 3$ & $\circ$ \\
\hline 55 & $\mathrm{M}_{11}$ & $\neq 2,3$ & & + \\
\hline 55 & $\mathrm{M}_{12}$ & $\neq 2,3$ & & + \\
\hline 55 & $\mathrm{M}_{22}$ & $\neq 2,7$ & & + \\
\hline 55 & $\mathrm{~J}_{1}$ & 19 & $b 5$ & + \\
\hline 55 & HS & 5 & & + \\
\hline 56 & $\mathfrak{A}_{8}$ & 0,7 & & + \\
\hline 56 & $2 . \mathfrak{A}_{8}$ & 0,7 & $z 3$ & $\circ$ \\
\hline 56 & $2 . \mathfrak{A}_{8}$ & 0,7 & $i 15$ & o \\
\hline 56 & $\mathfrak{A}_{9}$ & $\neq 2,3$ & & + \\
\hline 56 & $2 . \mathfrak{A}_{9}$ & $\neq 2,3$ & & + \\
\hline 56 & $\mathfrak{A}_{10}$ & 5 & & + \\
\hline 56 & 2. $\mathfrak{A}_{10}$ & 5 & $r 6, r 21$ & + \\
\hline 56 & $2 . \mathfrak{A}_{11}$ & 5 & $r 6, r 21$ & + \\
\hline 56 & $4_{1} \cdot \mathrm{L}_{3}(4)$ & $\neq 2,3$ & $i 1$ & $\circ$ \\
\hline 56 & $\mathrm{~L}_{3}(7)$ & 0,2 & & + \\
\hline 56 & $\mathrm{U}_{3}(8)$ & $\neq 2$ & & - \\
\hline 56 & 2. $\mathrm{U}_{4}(3)$ & $\neq 2,3$ & & + \\
\hline 56 & 2. $U_{6}(2)$ & $\neq 2$ & & + \\
\hline 56 & $\mathrm{~S}_{6}(2)$ & $\neq 2,3$ & & + \\
\hline 56 & $2 . \mathrm{S}_{6}(2)$ & 3 & $i 5$ & $\circ$ \\
\hline 56 & $2 . \mathrm{O}_{8}^{+}(2)$ & $\neq 2$ & & + \\
\hline
\end{tabular}


Corrigenda: Low-dimensional representations of quasi-simple groups

Table 2: Absolutely irreducible representations of quasi-simple groups, corrected

Continued from the previous page

\begin{tabular}{|c|c|c|c|c|}
\hline$d$ & $G$ & $\ell$ & field & ind \\
\hline 56 & 2.Sz(8) & $\neq 2,13$ & $c 13$ & + \\
\hline 56 & $2 . \mathrm{M}_{22}$ & $\neq 2,5$ & & + \\
\hline 56 & 4. $\mathrm{M}_{22}$ & $\neq 2$ & $z 8$ & ० \\
\hline 56 & $\mathrm{~J}_{1}$ & 2 & $b 5$ & - \\
\hline 56 & $\mathrm{~J}_{1}$ & 5 & & + \\
\hline 56 & $\mathrm{~J}_{1}$ & $\neq 5,19$ & $b 5$ & + \\
\hline 56 & $2 . J_{2}$ & 5 & & - \\
\hline 56 & $2 . \mathrm{J}_{2}$ & 0,7 & $b 5$ & - \\
\hline 56 & HS & 2 & & + \\
\hline 56 & 2.HS & $\neq 2,5$ & & + \\
\hline 57 & $\mathrm{~L}_{3}(7)$ & $\neq 2,7$ & & + \\
\hline 57 & $3 . \mathrm{L}_{3}(7)$ & $\neq 3,7$ & $z 3$ & $\circ$ \\
\hline 57 & $\mathrm{U}_{3}(8)$ & $\neq 2,3$ & $z 3$ & $\circ$ \\
\hline 57 & $3 . \mathrm{U}_{3}(8)$ & $\neq 2,3$ & $z 9$ & $\circ$ \\
\hline 57 & $\mathrm{~J}_{2}$ & 3 & $b 5$ & + \\
\hline 58 & $\mathrm{U}_{4}(2)$ & 5 & & + \\
\hline 58 & $2 . \mathrm{J}_{2}$ & 7 & $b 5$ & - \\
\hline 60 & $4_{2} \cdot \mathrm{L}_{3}(4)$ & 3 & $i 1, r 7$ & $\circ$ \\
\hline 60 & $6 . \mathrm{L}_{3}(4)$ & 0,5 & $z 3, b 7$ & $\circ$ \\
\hline 60 & $12_{2} \cdot \mathrm{L}_{3}(4)$ & 0,5 & $z 12, b 7$ & $\circ$ \\
\hline 60 & $\mathrm{U}_{4}(2)$ & 0,5 & & + \\
\hline 60 & 2. $\mathrm{U}_{4}(2)$ & 0,5 & & - \\
\hline 60 & 2. $\mathrm{U}_{4}(2)$ & 0,5 & $z 3$ & $\circ$ \\
\hline 60 & $\mathrm{U}_{5}(3)$ & $\neq 3$ & & - \\
\hline 60 & $\mathrm{~S}_{4}(11)$ & 2 & $b 11$ & $\circ$ \\
\hline 60 & $2 . \mathrm{S}_{4}(11)$ & $\neq 2,11$ & $b 11$ & $\circ$ \\
\hline 61 & $\mathrm{~L}_{6}(2)$ & 3,7 & & + \\
\hline 61 & $\mathrm{U}_{5}(3)$ & $\neq 2,3$ & $i 1$ & $\circ$ \\
\hline 61 & $\mathrm{~S}_{4}(11)$ & $\neq 2,11$ & $b 11$ & $\circ$ \\
\hline 62 & $\mathrm{~L}_{6}(2)$ & $0,5,31$ & & + \\
\hline 62 & $S_{6}(5)$ & $\neq 5$ & $b 5$ & - \\
\hline 63 & $\mathrm{~L}_{3}(4)$ & 5 & & + \\
\hline 63 & $\mathrm{~L}_{3}(4)$ & $\neq 2,5$ & $b 5$ & + \\
\hline 63 & $3 . \mathrm{L}_{3}(4)$ & 5 & $z 3$ & $\circ$ \\
\hline 63 & $3 . \mathrm{L}_{3}(4)$ & 0,7 & $z 3, b 5$ & ० \\
\hline 63 & $\mathrm{U}_{3}(4)$ & 13 & & + \\
\hline 63 & $2 . S_{6}(5)$ & $\neq 2,5$ & $b 5$ & + \\
\hline 63 & $\mathrm{Sz}(8)$ & 5 & & + \\
\hline 63 & $\mathrm{~J}_{2}$ & $\neq 2,5$ & & + \\
\hline 64 & $\mathfrak{A}_{8}$ & 0 & & + \\
\hline
\end{tabular}


Corrigenda: Low-dimensional representations of quasi-simple groups

Table 2: Absolutely irreducible representations of quasi-simple groups, corrected

Continued from the previous page

\begin{tabular}{|c|c|c|c|c|}
\hline$d$ & $G$ & $\ell$ & field & ind \\
\hline 64 & $2 . \mathfrak{A}_{8}$ & 0 & & - \\
\hline 64 & $\mathfrak{A}_{10}$ & 2 & & + \\
\hline 64 & $2 . \mathfrak{A}_{10}$ & 0,7 & & + \\
\hline 64 & $\mathfrak{A}_{13}$ & 2 & & - \\
\hline 64 & $\mathfrak{A}_{13}$ & 3 & & + \\
\hline 64 & $\mathfrak{A}_{14}$ & 2,3 & & + \\
\hline 64 & $2 . \mathfrak{A}_{14}$ & $\neq 2,7$ & & - \\
\hline 64 & $\mathfrak{A}_{15}$ & 2 & $b 15$ & $\circ$ \\
\hline 64 & $2 . \mathfrak{A}_{15}$ & 3,5 & & - \\
\hline 64 & $2 . \mathfrak{A}_{15}$ & $\neq 2,3,5$ & $b 15$ & $\circ$ \\
\hline 64 & $\mathfrak{A}_{16}$ & 2 & $b 7, b 15, b 39, b 55$ & $\circ$ \\
\hline 64 & $\mathrm{~L}_{3}(4)$ & 0 & & + \\
\hline 64 & 2. $\mathrm{L}_{3}(4)$ & 0,7 & & + \\
\hline 64 & $4_{1} \cdot \mathrm{L}_{3}(4)$ & 0,7 & $i 1$ & $\circ$ \\
\hline 64 & $4_{2} \cdot \mathrm{L}_{3}(4)$ & 0 & $i 1$ & $\circ$ \\
\hline 64 & $\mathrm{U}_{3}(4)$ & 0,3 & & + \\
\hline 64 & $\mathrm{U}_{4}(2)$ & 0 & & + \\
\hline 64 & 2. $\mathrm{U}_{4}(2)$ & 0 & & - \\
\hline 64 & $\mathrm{~S}_{4}(5)$ & 2 & & - \\
\hline 64 & $\mathrm{~S}_{4}(5)$ & 3 & & + \\
\hline 64 & $2 . S_{6}(2)$ & 5 & & + \\
\hline 64 & $2 . S_{6}(2)$ & 0,7 & $i 5$ & $\circ$ \\
\hline 64 & $\mathrm{Sz}(8)$ & 0,7 & & + \\
\hline 64 & 2.Sz(8) & 0,7 & & + \\
\hline 64 & $G_{2}(3)$ & $\neq 3$ & $z 3$ & $\circ$ \\
\hline 64 & $G_{2}(4)$ & 3 & & + \\
\hline 64 & 2. $\mathrm{M}_{22}$ & 11 & & + \\
\hline 64 & 4. $\mathrm{M}_{22}$ & 3 & $i 1$ & $\circ$ \\
\hline 64 & $\mathrm{~J}_{1}$ & 11 & $b 5, c 19$ & + \\
\hline 64 & $\mathbf{J}_{2}$ & 2 & $b 5$ & + \\
\hline 64 & $2 . \mathrm{J}_{2}$ & 5 & & - \\
\hline 64 & $2 . \mathrm{J}_{2}$ & 0 & $b 5$ & - \\
\hline 64 & Suz & 3 & & + \\
\hline 65 & $\mathfrak{A}_{13}$ & $\neq 2,3,11$ & & + \\
\hline 65 & $\mathrm{~L}_{4}(3)$ & $\neq 2,3$ & & + \\
\hline 65 & $\mathrm{U}_{3}(4)$ & 0,13 & $z 5$ & $\circ$ \\
\hline 65 & $\mathrm{U}_{3}(4)$ & $\neq 2,3$ & & + \\
\hline 65 & $\mathrm{~S}_{4}(5)$ & 0,13 & & + \\
\hline 65 & $\mathrm{Sz}(8)$ & $\neq 2,7$ & $y 7$ & + \\
\hline 65 & $G_{2}(4)$ & $\neq 2,3$ & & + \\
\hline
\end{tabular}


Corrigenda: Low-dimensional representations of quasi-simple groups

Table 2: Absolutely irreducible representations of quasi-simple groups, corrected

Continued from the previous page

\begin{tabular}{|c|c|c|c|c|}
\hline$d$ & $G$ & $\ell$ & field & ind \\
\hline 66 & $\mathfrak{A}_{10}$ & 7 & & + \\
\hline 66 & $\mathfrak{A}_{11}$ & 7 & & + \\
\hline 66 & $\mathfrak{A}_{13}$ & $\neq 2,13$ & & + \\
\hline 66 & $\mathfrak{A}_{14}$ & 7 & & + \\
\hline 66 & $\mathrm{U}_{5}(2)$ & $\neq 2,3$ & $z 3$ & $\circ$ \\
\hline 66 & $\mathrm{M}_{12}$ & $\neq 2,3$ & & + \\
\hline 66 & 6. $\mathrm{M}_{22}$ & 7 & $z 3$ & ○ \\
\hline 66 & 6. $\mathrm{M}_{22}$ & $0,5,11$ & $z 3, b 7$ & $\circ$ \\
\hline 66 & 3.Suz & $\neq 3$ & $z 3$ & ○ \\
\hline 69 & $\mathrm{~J}_{1}$ & 11 & $b 5, c 19$ & + \\
\hline 70 & $\mathfrak{A}_{8}$ & $\neq 2,3$ & & + \\
\hline 70 & $2 . \mathrm{L}_{3}(4)$ & $\neq 2,3$ & & + \\
\hline 70 & $\mathrm{U}_{4}(3)$ & 2 & $z 3$ & ○ \\
\hline 70 & 2. $\mathrm{U}_{4}(3)$ & $\neq 2,3$ & & + \\
\hline 70 & 2. $\mathrm{U}_{4}(3)$ & $\neq 2,3$ & $z 3$ & ० \\
\hline 70 & $\mathrm{~S}_{6}(2)$ & $\neq 2,3$ & & + \\
\hline 70 & $\mathrm{M}_{22}$ & 2 & $b 11$ & ○ \\
\hline 70 & $\mathrm{~J}_{2}$ & 5 & & + \\
\hline 70 & $\mathbf{J}_{2}$ & 0,7 & $b 5$ & + \\
\hline 71 & $\mathrm{~L}_{3}(8)$ & 73 & & + \\
\hline 72 & $2 . \mathfrak{A}_{9}$ & 7 & $z 3, r 2$ & o \\
\hline 72 & $\mathrm{~L}_{3}(8)$ & $\neq 2,73$ & & + \\
\hline 72 & $\mathrm{U}_{3}(9)$ & $\neq 3$ & & - \\
\hline 73 & $\mathrm{~L}_{3}(8)$ & $\neq 2,7$ & $z 7$ & ○ \\
\hline 73 & $\mathrm{U}_{3}(9)$ & $\neq 2,3$ & & + \\
\hline 73 & $\mathrm{U}_{3}(9)$ & $\neq 3,5$ & $z 5$ & $\circ$ \\
\hline 75 & $\mathfrak{A}_{10}$ & 0,5 & & + \\
\hline 75 & $\mathrm{U}_{3}(4)$ & $\neq 2,13$ & $d 13$ & ○ \\
\hline 75 & $\mathrm{~J}_{1}$ & 7 & & + \\
\hline 76 & $\mathfrak{A}_{14}$ & 13 & & + \\
\hline 76 & $\mathfrak{A}_{15}$ & 13 & & + \\
\hline 76 & $\mathrm{~J}_{1}$ & 2 & & - \\
\hline 76 & $\mathrm{~J}_{1}$ & $\neq 7,11$ & & + \\
\hline 77 & $\mathfrak{A}_{14}$ & $\neq 2,3,13$ & & + \\
\hline 77 & ${ }^{2} F_{4}(2){ }^{\prime}$ & 3 & & + \\
\hline 77 & $\mathrm{~J}_{1}$ & $\neq 2,3,19$ & & + \\
\hline 77 & $\mathrm{~J}_{1}$ & $\neq 2,5$ & $b 5$ & + \\
\hline 77 & HS & $\neq 2,5$ & & + \\
\hline 77 & $\mathrm{Fi}_{22}$ & 3 & & + \\
\hline 78 & $\mathfrak{A}_{9}$ & 2 & & + \\
\hline
\end{tabular}


Corrigenda: Low-dimensional representations of quasi-simple groups

Table 2: Absolutely irreducible representations of quasi-simple groups, corrected

Continued from the previous page

\begin{tabular}{|c|c|c|c|c|}
\hline$d$ & $G$ & $\ell$ & field & ind \\
\hline 78 & $\mathfrak{A}_{14}$ & $\neq 2,7$ & & + \\
\hline 78 & $\mathfrak{A}_{15}$ & 3,5 & & + \\
\hline 78 & $6_{1} \cdot \mathrm{U}_{4}(3)$ & 5 & $z 3$ & $\circ$ \\
\hline 78 & $\mathrm{~S}_{4}(5)$ & $\neq 2,5$ & $b 5$ & + \\
\hline 78 & $\mathrm{~S}_{6}(3)$ & $\neq 3$ & & + \\
\hline 78 & $\mathrm{O}_{7}(3)$ & $\neq 3$ & & + \\
\hline 78 & $G_{2}(3)$ & $\neq 3$ & & + \\
\hline 78 & $G_{2}(4)$ & $\neq 2$ & & + \\
\hline 78 & ${ }^{2} F_{4}(2)^{\prime}$ & $\neq 2,3$ & & + \\
\hline 78 & $\mathrm{M}_{12}$ & 5 & & + \\
\hline 78 & $3 . \mathrm{M}_{22}$ & 5 & $z 3$ & $\circ$ \\
\hline 78 & $\mathrm{~J}_{3}$ & 2 & $b 5, b 17$ & + \\
\hline 78 & Suz & 3 & & + \\
\hline 78 & 3.Suz & $\neq 2,3$ & $z 3$ & $\circ$ \\
\hline 78 & $\mathrm{Fi}_{22}$ & 2 & & + \\
\hline 78 & $\mathrm{Fi}_{22}$ & $\neq 2,3$ & & + \\
\hline 80 & $4_{1} \cdot \mathrm{L}_{3}(4)$ & 0,5 & $i 1, r 7$ & $\circ$ \\
\hline 80 & $4_{2} \cdot \mathrm{L}_{3}(4)$ & 0,5 & $i 1, r 7$ & ० \\
\hline 80 & 2. $\mathrm{U}_{4}(2)$ & 0,5 & & - \\
\hline 80 & $\mathrm{~J}_{3}$ & 2 & $b 17$ & + \\
\hline 81 & $\mathrm{U}_{4}(2)$ & 0 & & + \\
\hline 83 & $\mathfrak{A}_{9}$ & 5 & & + \\
\hline 83 & $\mathrm{~L}_{4}(4)$ & 5,17 & & + \\
\hline 83 & $\mathrm{~S}_{6}(2)$ & 5 & & + \\
\hline 83 & $\mathrm{O}_{8}^{+}(2)$ & 5 & & + \\
\hline 83 & $\mathrm{O}_{8}^{-}(2)$ & 17 & & + \\
\hline 84 & $\mathfrak{A}_{9}$ & 0,7 & & + \\
\hline 84 & $\mathfrak{A}_{10}$ & $\neq 2,5$ & & + \\
\hline 84 & $\mathfrak{A}_{11}$ & 11 & & + \\
\hline 84 & $3 . \mathrm{L}_{3}(4)$ & 0,7 & $z 3$ & $\circ$ \\
\hline 84 & $12_{2} \cdot \mathrm{L}_{3}(4)$ & 0,7 & $z 12$ & $\circ$ \\
\hline 84 & $\mathrm{~L}_{4}(4)$ & $0,3,7$ & & + \\
\hline 84 & $\mathrm{U}_{3}(5)$ & $\neq 2,5$ & & + \\
\hline 84 & $3 . U_{3}(5)$ & $\neq 3,5$ & $z 3$ & $\circ$ \\
\hline 84 & $3{ }_{1} \cdot \mathrm{U}_{4}(3)$ & 2 & $z 3$ & $\circ$ \\
\hline 84 & $6_{1} \cdot \mathrm{U}_{4}(3)$ & 0,7 & $z 3$ & ○ \\
\hline 84 & $12_{1} \cdot \mathrm{U}_{4}(3)$ & $\neq 2,3$ & $z 12$ & $\circ$ \\
\hline 84 & $\mathrm{~S}_{4}(13)$ & 2 & $b 13$ & - \\
\hline 84 & $2 . \mathrm{S}_{4}(13)$ & $\neq 2,13$ & $b 13$ & - \\
\hline 84 & $S_{6}(2)$ & 0,7 & & + \\
\hline
\end{tabular}


Corrigenda: Low-dimensional representations of quasi-simple groups

Table 2: Absolutely irreducible representations of quasi-simple groups, corrected

Continued from the previous page

\begin{tabular}{|c|c|c|c|c|}
\hline$d$ & $G$ & $\ell$ & field & ind \\
\hline 84 & $\mathrm{O}_{8}^{+}(2)$ & 0,7 & & + \\
\hline 84 & $\mathrm{O}_{8}^{-}(2)$ & $0,5,7$ & & + \\
\hline 84 & 2. $\mathrm{M}_{12}$ & 3 & $i 5, b 11$ & $\circ$ \\
\hline 84 & $3 . \mathrm{M}_{22}$ & 11 & $z 3$ & ○ \\
\hline 84 & $3 . \mathrm{M}_{22}$ & 2 & $z 3, b 11$ & $\circ$ \\
\hline 84 & $\mathrm{~J}_{2}$ & 2 & & + \\
\hline 84 & $2 . \mathrm{J}_{2}$ & 0,7 & & - \\
\hline 84 & $\mathrm{~J}_{3}$ & 2,3 & $b 19$ & o \\
\hline 85 & $\mathrm{~L}_{4}(4)$ & $\neq 2,3$ & $z 3$ & o \\
\hline 85 & $\mathrm{U}_{8}(2)$ & 3 & & + \\
\hline 85 & $\mathrm{U}_{8}(2)$ & $\neq 2,3$ & $z 3$ & ० \\
\hline 85 & $\mathrm{~S}_{4}(4)$ & $\neq 2,3$ & & + \\
\hline 85 & $\mathrm{~S}_{4}(13)$ & $\neq 2,13$ & $b 13$ & + \\
\hline 85 & $\mathrm{~S}_{8}(2)$ & $\neq 2,3$ & & + \\
\hline 85 & $\mathbf{J}_{2}$ & 5 & & + \\
\hline 85 & $\mathrm{~J}_{3}$ & 19 & & + \\
\hline 85 & $\mathrm{~J}_{3}$ & $0,5,17$ & $b 19$ & ० \\
\hline 86 & $\mathrm{U}_{8}(2)$ & $\neq 2,3$ & & + \\
\hline 88 & 4. $\mathrm{M}_{22}$ & 5 & $z 8, b 7$ & $\circ$ \\
\hline 89 & $\mathfrak{A}_{10}$ & 7 & & + \\
\hline 89 & $\mathfrak{A}_{11}$ & 5 & & + \\
\hline 89 & $\mathfrak{A}_{12}$ & 5 & & + \\
\hline 89 & $\mathfrak{A}_{15}$ & 7 & & + \\
\hline 89 & $\mathfrak{A}_{16}$ & 7 & & + \\
\hline 89 & $\mathrm{~L}_{3}(9)$ & 7,13 & & + \\
\hline 89 & $\mathrm{~L}_{4}(3)$ & 13 & & + \\
\hline 89 & $\mathrm{U}_{4}(3)$ & 7 & & + \\
\hline 89 & $\mathrm{~S}_{4}(5)$ & 13 & & + \\
\hline 89 & $\mathrm{~J}_{1}$ & 7 & $c 19$ & + \\
\hline 89 & $\mathrm{~J}_{2}$ & 7 & & + \\
\hline 90 & $\mathfrak{A}_{10}$ & 0,3 & & + \\
\hline 90 & $\mathfrak{A}_{15}$ & $\neq 7,13$ & & + \\
\hline 90 & $\mathfrak{A}_{16}$ & 2 & & + \\
\hline 90 & $2 . \mathrm{L}_{3}(4)$ & $\neq 2,7$ & & + \\
\hline 90 & $6 . \mathrm{L}_{3}(4)$ & 0,5 & $z 3$ & ० \\
\hline 90 & $\mathrm{~L}_{3}(9)$ & $0,2,5$ & & + \\
\hline 90 & $\mathrm{~L}_{4}(3)$ & 0,5 & & + \\
\hline 90 & $\mathrm{U}_{4}(3)$ & 0,5 & & + \\
\hline 90 & $3_{1} \cdot \mathrm{U}_{4}(3)$ & 2 & $z 3$ & o \\
\hline 90 & $6_{2} \cdot \mathrm{U}_{4}(3)$ & $\neq 2,3$ & $z 3$ & $\circ$ \\
\hline
\end{tabular}


Corrigenda: Low-dimensional representations of quasi-simple groups

Table 2: Absolutely irreducible representations of quasi-simple groups, corrected

Continued from the previous page

\begin{tabular}{|c|c|c|c|c|}
\hline$d$ & $G$ & $\ell$ & field & ind \\
\hline 90 & $\mathrm{~S}_{4}(5)$ & 0,3 & & + \\
\hline 90 & $\mathrm{O}_{7}(3)$ & 2 & & + \\
\hline 90 & $G_{2}(3)$ & 2 & & + \\
\hline 90 & 6. $\mathrm{M}_{22}$ & 11 & $z 12$ & $\circ$ \\
\hline 90 & $\mathrm{~J}_{2}$ & $\neq 2,7$ & & + \\
\hline 91 & $\mathfrak{A}_{15}$ & $\neq 2,3,5$ & & + \\
\hline 91 & $\mathrm{~L}_{3}(9)$ & $\neq 2,3$ & & + \\
\hline 91 & $\mathrm{~L}_{3}(9)$ & $\neq 2,3$ & $i 1$ & $\circ$ \\
\hline 91 & $\mathrm{~L}_{3}(9)$ & $\neq 2,3$ & $z 8$ & $\circ$ \\
\hline 91 & $\mathrm{~S}_{6}(2)$ & 3 & & + \\
\hline 91 & $\mathrm{~S}_{6}(3)$ & $\neq 2,3$ & $z 3$ & ० \\
\hline 91 & $\mathrm{O}_{7}(3)$ & $\neq 2,3$ & & + \\
\hline 91 & $\mathrm{Sz}(8)$ & $\neq 2,5$ & & + \\
\hline 91 & $G_{2}(3)$ & $\neq 2,3$ & & + \\
\hline 91 & $\mathrm{M}_{12}$ & 11 & & + \\
\hline 92 & $2 . G_{2}(4)$ & 5 & & - \\
\hline 94 & $\mathrm{~L}_{5}(2)$ & 7 & & + \\
\hline 94 & $\mathrm{~S}_{6}(2)$ & 7 & & + \\
\hline 96 & $\mathrm{~L}_{3}(5)$ & 31 & & + \\
\hline 96 & $\mathrm{~L}_{3}(5)$ & $\neq 5,31$ & $x 31$ & $\circ$ \\
\hline 96 & $\mathrm{~L}_{3}(7)$ & 3 & & + \\
\hline 96 & $3 . \mathrm{L}_{3}(7)$ & $\neq 3,7$ & $z 3$ & $\circ$ \\
\hline 96 & $12 . \mathrm{M}_{22}$ & 11 & $z 12$ & ○ \\
\hline 98 & $\mathrm{~S}_{6}(2)$ & 3 & & + \\
\hline 98 & $\mathrm{M}_{12}$ & 5 & & + \\
\hline 98 & $\mathrm{M}_{22}$ & 2 & & - \\
\hline 98 & $\mathrm{M}_{22}$ & 5 & & + \\
\hline 98 & HS & 5 & & + \\
\hline 99 & $\mathrm{M}_{12}$ & $\neq 2,5$ & & + \\
\hline 99 & $\mathrm{M}_{22}$ & $0,3,11$ & & + \\
\hline 99 & 3. $\mathrm{M}_{22}$ & $0,7,11$ & $z 3$ & $\circ$ \\
\hline 100 & $\mathfrak{A}_{11}$ & 2 & & + \\
\hline 100 & $\mathfrak{A}_{12}$ & 2 & & + \\
\hline 100 & $\mathrm{U}_{5}(2)$ & 3 & & + \\
\hline 101 & $\mathfrak{A}_{9}$ & 7 & & + \\
\hline 101 & $\mathfrak{A}_{10}$ & 7 & & + \\
\hline 101 & $\mathbf{J}_{2}$ & 7 & & + \\
\hline 101 & $\mathrm{He}$ & 2 & $b 7$ & $\circ$ \\
\hline 103 & $\mathfrak{A}_{16}$ & 3,5 & & + \\
\hline 103 & $\mathfrak{A}_{17}$ & 3,5 & & + \\
\hline
\end{tabular}


Corrigenda: Low-dimensional representations of quasi-simple groups

Table 2: Absolutely irreducible representations of quasi-simple groups, corrected

Continued from the previous page

\begin{tabular}{|c|c|c|c|c|}
\hline$d$ & $G$ & $\ell$ & field & ind \\
\hline 103 & $G_{2}(3)$ & 7 & & + \\
\hline 104 & 2.: $\mathfrak{A}_{9}$ & 3 & & + \\
\hline 104 & $\mathfrak{A}_{16}$ & $0,11,13$ & & + \\
\hline 104 & $\mathrm{U}_{3}(5)$ & 2 & & - \\
\hline 104 & $\mathrm{U}_{4}(5)$ & 2 & & - \\
\hline 104 & $\mathrm{U}_{4}(5)$ & 3 & & + \\
\hline 104 & $\mathrm{U}_{4}(5)$ & $\neq 3,5$ & $z 3$ & $\circ$ \\
\hline 104 & 2. $\mathrm{U}_{4}(5)$ & $0,7,13$ & $z 3$ & ० \\
\hline 104 & 2. $\mathrm{U}_{4}(5)$ & $\neq 2,5$ & & - \\
\hline 104 & $\mathrm{~S}_{4}(5)$ & $\neq 5$ & & + \\
\hline 104 & $2 . \mathrm{S}_{4}(5)$ & 0,13 & & - \\
\hline 104 & $2 . \mathrm{S}_{6}(2)$ & 3,5 & & + \\
\hline 104 & $\mathrm{O}_{7}(3)$ & 2 & & + \\
\hline 104 & $2 . \mathrm{O}_{8}^{+}(2)$ & 3,5 & & + \\
\hline 104 & 2.Sz(8) & 0,13 & & + \\
\hline 104 & $G_{2}(3)$ & 0,13 & & + \\
\hline 104 & $2 . G_{2}(4)$ & $\neq 2,5$ & $b 5$ & - \\
\hline 104 & $\mathrm{M}_{23}$ & 3 & $b 11, b 23$ & ० \\
\hline 104 & $\mathrm{McL}$ & 3 & $b 11$ & $\circ$ \\
\hline 104 & $\mathrm{He}$ & 5 & $r 21$ & + \\
\hline 105 & $\mathfrak{A}_{9}$ & $\neq 2,3$ & & + \\
\hline 105 & $\mathfrak{A}_{16}$ & $\neq 2$ & & + \\
\hline 105 & $\mathfrak{A}_{17}$ & 17 & & + \\
\hline 105 & $\mathrm{U}_{3}(5)$ & 0,7 & & + \\
\hline 105 & $3 . U_{3}(5)$ & 0,7 & $z 3$ & ○ \\
\hline 105 & $3_{1} . \mathrm{U}_{4}(3)$ & $\neq 2,3$ & $z 3$ & o \\
\hline 105 & $\mathrm{U}_{4}(5)$ & $0,7,13$ & & + \\
\hline 105 & $\mathrm{~S}_{6}(2)$ & $\neq 2,3$ & & + \\
\hline 105 & $\mathrm{~S}_{6}(3)$ & $\neq 2,3$ & & + \\
\hline 105 & $\mathrm{O}_{7}(3)$ & $\neq 2,3$ & & + \\
\hline 105 & $3 . \mathrm{M}_{22}$ & $0,5,7$ & $z 3, b 11$ & ० \\
\hline 106 & $\mathrm{~J}_{1}$ & 11 & $b 5, c 19$ & + \\
\hline 108 & 2. $\mathrm{M}_{12}$ & 11 & & + \\
\hline 109 & $\mathfrak{A}_{11}$ & 3 & & + \\
\hline 109 & ${ }^{2} F_{4}(2)^{\prime}$ & 5 & $r 2, r 3, b 13$ & + \\
\hline 110 & $\mathfrak{A}_{11}$ & $0,5,11$ & & + \\
\hline 110 & $\mathrm{U}_{3}(11)$ & $\neq 11$ & & - \\
\hline 110 & $\mathrm{U}_{5}(2)$ & $\neq 2$ & & - \\
\hline 110 & $\mathrm{U}_{5}(2)$ & $\neq 2,3$ & $z 3$ & $\circ$ \\
\hline 110 & 2. $\mathrm{M}_{12}$ & $\neq 2,3$ & $i 2$ & ० \\
\hline
\end{tabular}


Corrigenda: Low-dimensional representations of quasi-simple groups

Table 2: Absolutely irreducible representations of quasi-simple groups, corrected

Continued from the previous page

\begin{tabular}{|c|c|c|c|c|}
\hline$d$ & $G$ & $\ell$ & field & ind \\
\hline 110 & $\mathrm{~J}_{3}$ & 19 & $b 17, y 9$ & + \\
\hline 110 & Suz & 2 & $b 5, b 13, r 21$ & + \\
\hline 111 & $\mathrm{U}_{3}(11)$ & $\neq 2,11$ & & + \\
\hline 111 & $\mathrm{U}_{3}(11)$ & $\neq 2,11$ & $i 1$ & $\circ$ \\
\hline 111 & 3. $\mathrm{U}_{3}(11)$ & $\neq 3,11$ & $z 3$ & $\circ$ \\
\hline 111 & 3. $\mathrm{U}_{3}(11)$ & $0,5,37$ & $z 12$ & ० \\
\hline 111 & Ly & 5 & & + \\
\hline 112 & 2.: $\mathfrak{A}_{9}$ & 0,7 & & + \\
\hline 112 & $2 . S_{6}(2)$ & 0,7 & & + \\
\hline 112 & $2 . \mathrm{O}_{8}^{+}(2)$ & 0,7 & & + \\
\hline 112 & $\mathrm{~J}_{4}$ & 2 & & + \\
\hline 114 & $6_{1} \cdot \mathrm{U}_{4}(3)$ & 7 & $z 3$ & $\circ$ \\
\hline 115 & $\mathfrak{A}_{9}$ & 7 & & + \\
\hline 118 & $\mathfrak{A}_{17}$ & 2 & & + \\
\hline 118 & $\mathfrak{A}_{18}$ & 2 & & + \\
\hline 118 & $\mathrm{~S}_{8}(2)$ & 3,5 & & + \\
\hline 119 & $\mathfrak{A}_{17}$ & $\neq 2,3,5$ & & + \\
\hline 119 & $\mathrm{~L}_{5}(3)$ & 11 & & + \\
\hline 119 & $\mathrm{U}_{5}(2)$ & 11 & & + \\
\hline 119 & $\mathrm{~S}_{8}(2)$ & $0,7,17$ & & + \\
\hline 119 & $\mathrm{~J}_{1}$ & 11 & $c 19$ & + \\
\hline 120 & $\mathfrak{A}_{9}$ & 0,5 & & + \\
\hline 120 & $2 . \mathfrak{A}_{9}$ & 0,5 & $z 3$ & $\circ$ \\
\hline 120 & $\mathfrak{A}_{11}$ & $\neq 2,11$ & & + \\
\hline 120 & $\mathfrak{A}_{12}$ & 3 & & + \\
\hline 120 & $\mathfrak{A}_{17}$ & $\neq 2,17$ & & + \\
\hline 120 & $\mathfrak{A}_{18}$ & 3 & & + \\
\hline 120 & $12_{1} \cdot \mathrm{L}_{3}(4)$ & 0,5 & $z 12$ & ○ \\
\hline 120 & $\mathrm{~L}_{5}(3)$ & $\neq 3,11$ & & + \\
\hline 120 & $\mathrm{U}_{4}(3)$ & 2 & & + \\
\hline 120 & 2. $U_{4}(3)$ & $\neq 2,3$ & & + \\
\hline 120 & 4. $U_{4}(3)$ & $\neq 2,3$ & $i 1$ & $\circ$ \\
\hline 120 & $6_{1} \cdot \mathrm{U}_{4}(3)$ & 0,5 & $z 3$ & ० \\
\hline 120 & $12_{1} \cdot \mathrm{U}_{4}(3)$ & $\neq 2,3$ & $z 12$ & ○ \\
\hline 120 & $\mathrm{U}_{5}(2)$ & 0,5 & & + \\
\hline 120 & 2. $\mathrm{U}_{6}(2)$ & 3 & & + \\
\hline 120 & 6. $U_{6}(2)$ & $\neq 2,3$ & $z 3$ & ० \\
\hline 120 & $\mathrm{~S}_{6}(2)$ & 0,5 & & + \\
\hline 120 & $2 . S_{6}(2)$ & $\neq 2,3$ & & + \\
\hline 120 & $\mathrm{M}_{12}$ & 0,5 & & + \\
\hline
\end{tabular}


Corrigenda: Low-dimensional representations of quasi-simple groups

Table 2: Absolutely irreducible representations of quasi-simple groups, corrected

Continued from the previous page

\begin{tabular}{|c|c|c|c|c|}
\hline$d$ & $G$ & $\ell$ & field & ind \\
\hline 120 & $2 . \mathrm{M}_{12}$ & 0,5 & & + \\
\hline 120 & 2. $\mathrm{M}_{22}$ & $\neq 2,11$ & & + \\
\hline 120 & 6. $\mathrm{M}_{22}$ & $0,5,11$ & $z 3$ & $\circ$ \\
\hline 120 & $12 . \mathrm{M}_{22}$ & $\neq 2,3$ & $z 24$ & ० \\
\hline 120 & $\mathrm{M}_{23}$ & 2 & & + \\
\hline 120 & $\mathrm{M}_{24}$ & 2 & & + \\
\hline 120 & $\mathrm{~J}_{1}$ & $\neq 11,19$ & $c 19$ & + \\
\hline 120 & 2.HS & 5 & $i 1$ & $\circ$ \\
\hline 121 & $\mathrm{~L}_{5}(3)$ & $\neq 2,3$ & & + \\
\hline 121 & $\mathrm{~S}_{10}(3)$ & $\neq 3$ & $z 3$ & $\circ$ \\
\hline 122 & 2. $\mathrm{S}_{10}(3)$ & $\neq 2,3$ & $z 3$ & ○ \\
\hline 123 & $\mathrm{~L}_{5}(2)$ & 5 & & + \\
\hline 124 & $\mathfrak{A}_{10}$ & 7 & & + \\
\hline 124 & $\mathrm{~L}_{3}(5)$ & $\neq 5$ & & + \\
\hline 124 & $\mathrm{~L}_{3}(5)$ & 2 & & - \\
\hline 124 & $\mathrm{~L}_{3}(5)$ & $\neq 2,5$ & $i 1$ & o \\
\hline 124 & $\mathrm{~L}_{3}(5)$ & 0,31 & $y 24^{\prime}$ & ○ \\
\hline 124 & $\mathrm{~L}_{5}(2)$ & $0,3,31$ & & + \\
\hline 124 & $\mathrm{U}_{3}(5)$ & 7 & & + \\
\hline 124 & $\mathrm{Sz}(32)$ & $\neq 2$ & $i 1$ & $\circ$ \\
\hline 124 & $G_{2}(5)$ & $\neq 5$ & & + \\
\hline 124 & ${ }^{2} F_{4}(2)^{\prime}$ & 3 & $b 13$ & + \\
\hline 124 & $\mathbf{J}_{2}$ & 7 & & + \\
\hline 125 & $\mathrm{~L}_{3}(5)$ & 0 & & + \\
\hline 125 & $\mathrm{~L}_{7}(2)$ & 127 & & + \\
\hline 125 & $\mathrm{U}_{3}(5)$ & 0 & & + \\
\hline 126 & $\mathfrak{A}_{10}$ & $\neq 2,5$ & & + \\
\hline 126 & $\mathfrak{A}_{11}$ & 11 & & + \\
\hline 126 & $\mathfrak{A}_{11}$ & $\neq 2,11$ & $b 11$ & o \\
\hline 126 & $\mathfrak{A}_{12}$ & 3 & $b 11, i 35$ & $\circ$ \\
\hline 126 & $\mathrm{~L}_{7}(2)$ & $\neq 2,127$ & & + \\
\hline 126 & $\mathrm{U}_{3}(5)$ & $\neq 2,5$ & & + \\
\hline 126 & $\mathrm{U}_{3}(5)$ & $\neq 2,5$ & $i 2$ & $\circ$ \\
\hline 126 & $3 . U_{3}(5)$ & 0,7 & $z 3$ & ○ \\
\hline 126 & $3 . U_{3}(5)$ & 0,7 & $z 3, i 2$ & ० \\
\hline 126 & $3_{2} \cdot \mathrm{U}_{4}(3)$ & $\neq 2,3$ & $z 3$ & $\circ$ \\
\hline 126 & $6_{1} \cdot U_{4}(3)$ & $\neq 2,3$ & $z 3$ & ○ \\
\hline 126 & $6_{2} \cdot \mathrm{U}_{4}(3)$ & $\neq 2,3$ & $z 3$ & ० \\
\hline 126 & $6_{2} \cdot U_{4}(3)$ & $\neq 2,3$ & $z 12$ & $\circ$ \\
\hline 126 & $\mathrm{~S}_{4}(7)$ & 2 & & + \\
\hline
\end{tabular}


Corrigenda: Low-dimensional representations of quasi-simple groups

Table 2: Absolutely irreducible representations of quasi-simple groups, corrected

Continued from the previous page

\begin{tabular}{|c|c|c|c|c|}
\hline$d$ & $G$ & $\ell$ & field & ind \\
\hline 126 & $\mathrm{~S}_{4}(7)$ & $\neq 2,7$ & & + \\
\hline 126 & $2 . \mathrm{M}_{22}$ & 11 & & + \\
\hline 126 & 2. $\mathrm{M}_{22}$ & $\neq 2,11$ & $b 11$ & $\circ$ \\
\hline 126 & $6 . \mathrm{M}_{22}$ & $0,5,7$ & $z 3, b 11$ & $\circ$ \\
\hline 126 & $\mathrm{~J}_{2}$ & 0,7 & & + \\
\hline 126 & $2 . \mathrm{J}_{2}$ & $\neq 2,5$ & $b 5$ & - \\
\hline 126 & $3 . \mathrm{J}_{3}$ & 2 & $z 3, b 17, b 19$ & ○ \\
\hline 126 & 3.McL & 11 & $z 3$ & $\circ$ \\
\hline 126 & 3.McL & $\neq 3,11$ & $z 3, b 11$ & $\circ$ \\
\hline 126 & $\mathrm{Co}_{3}$ & 3 & $i 5, b 11, b 23$ & ० \\
\hline 128 & $2 . \mathfrak{A}_{11}$ & 11 & & + \\
\hline 128 & $2 . \mathfrak{A}_{12}$ & 11 & & + \\
\hline 128 & $2 . \mathfrak{A}_{16}$ & $\neq 2$ & & + \\
\hline 128 & $\mathfrak{A}_{17}$ & 2 & $b 17$ & + \\
\hline 128 & $2 . \mathfrak{A}_{17}$ & 17 & & + \\
\hline 128 & $2 . \mathfrak{A}_{17}$ & $\neq 2,17$ & $b 17$ & + \\
\hline 128 & $2 . \mathfrak{A}_{18}$ & 3 & $r 2, r 5, r 14, b 17, b 65, b 77$ & + \\
\hline 130 & $\mathrm{~S}_{4}(5)$ & 0,13 & & + \\
\hline 131 & $\mathfrak{A}_{11}$ & 3,7 & & + \\
\hline 131 & $\mathfrak{A}_{12}$ & 3,7 & & + \\
\hline 131 & $\mathrm{~L}_{3}(11)$ & 7,19 & & + \\
\hline 132 & $\mathfrak{A}_{11}$ & 0,11 & & + \\
\hline 132 & $\mathfrak{A}_{12}$ & 0,11 & & + \\
\hline 132 & $\mathrm{~L}_{3}(11)$ & $0,2,3,5$ & & + \\
\hline 132 & $12_{1} \cdot \mathrm{U}_{4}(3)$ & 5 & $z 12$ & $\circ$ \\
\hline 132 & HS & 2 & & - \\
\hline 132 & $\mathrm{HN}$ & 2 & $b 5$ & - \\
\hline 133 & $\mathfrak{A}_{9}$ & 5 & & + \\
\hline 133 & $\mathfrak{A}_{10}$ & 5 & $r 21$ & + \\
\hline 133 & $\mathfrak{A}_{11}$ & 5 & $r 21$ & + \\
\hline 133 & $\mathrm{~L}_{3}(11)$ & $\neq 2,11$ & & + \\
\hline 133 & $\mathrm{~L}_{3}(11)$ & $\neq 5,11$ & $z 5$ & $\circ$ \\
\hline 133 & $\mathrm{U}_{3}(8)$ & $\neq 2$ & & + \\
\hline 133 & $\mathrm{~S}_{6}(2)$ & 5 & & + \\
\hline 133 & $\mathrm{M}_{22}$ & 5 & & + \\
\hline 133 & $\mathrm{~J}_{1}$ & $\neq 2,11$ & & + \\
\hline 133 & $\mathrm{~J}_{1}$ & $0,7,19$ & $b 5$ & + \\
\hline 133 & $\mathbf{J}_{2}$ & 3 & & + \\
\hline 133 & HS & 5 & & + \\
\hline 133 & $\mathrm{Ru}$ & 5 & & + \\
\hline
\end{tabular}


Corrigenda: Low-dimensional representations of quasi-simple groups

Table 2: Absolutely irreducible representations of quasi-simple groups, corrected

Continued from the previous page

\begin{tabular}{|c|c|c|c|c|}
\hline$d$ & $G$ & $\ell$ & field & ind \\
\hline 133 & $\mathrm{HN}$ & 5 & & + \\
\hline 133 & $\mathrm{HN}$ & $\neq 2,5$ & $b 5$ & + \\
\hline 134 & $\mathfrak{A}_{9}$ & 5 & & + \\
\hline 134 & $\mathfrak{A}_{18}$ & 17 & & + \\
\hline 134 & $\mathfrak{A}_{19}$ & 17 & & + \\
\hline 134 & $\mathrm{~S}_{8}(2)$ & 17 & & + \\
\hline 135 & $\mathfrak{A}_{18}$ & $\neq 2,17$ & & + \\
\hline 135 & $\mathrm{~S}_{8}(2)$ & $\neq 2,17$ & & + \\
\hline 136 & $\mathfrak{A}_{18}$ & $\neq 2,3$ & & + \\
\hline 136 & $\mathfrak{A}_{19}$ & 19 & & + \\
\hline 140 & $\mathrm{U}_{4}(3)$ & $\neq 2,3$ & & + \\
\hline 140 & 4. $\mathrm{U}_{4}(3)$ & $\neq 2,3$ & $i 1$ & $\circ$ \\
\hline 141 & $\mathrm{~S}_{6}(2)$ & 5 & & + \\
\hline 142 & Suz & 2 & & + \\
\hline 143 & $\mathfrak{A}_{12}$ & 3 & & + \\
\hline 143 & $\mathfrak{A}_{13}$ & 3 & & + \\
\hline 143 & Suz & $\neq 2,3$ & & + \\
\hline 144 & $\mathfrak{A}_{11}$ & 2 & & + \\
\hline 144 & $2 . \mathfrak{A}_{11}$ & $0,3,7$ & & + \\
\hline 144 & $\mathfrak{A}_{12}$ & 5 & & + \\
\hline 144 & $\mathfrak{A}_{12}$ & 2 & $i 35, z 3$ & ० \\
\hline 144 & $2 . \mathfrak{A}_{12}$ & 3 & $i 2, i 5, r 7$ & o \\
\hline 144 & $\mathfrak{A}_{13}$ & 5 & & + \\
\hline 144 & $\mathfrak{A}_{13}$ & 2 & $i 35, z 3$ & ० \\
\hline 144 & $2 . \mathfrak{A}_{13}$ & 3 & $i 2, i 5, r 7$ & $\circ$ \\
\hline 144 & $\mathrm{U}_{3}(5)$ & $\neq 5,7$ & $b 7$ & $\circ$ \\
\hline 144 & $3 . U_{3}(5)$ & 0,2 & $z 3, b 7$ & ○ \\
\hline 144 & $\mathrm{~S}_{4}(17)$ & 2 & $b 17$ & - \\
\hline 144 & $2 . S_{4}(17)$ & $\neq 2,17$ & $b 17$ & - \\
\hline 144 & $\mathrm{M}_{12}$ & 0,2 & & + \\
\hline 144 & 4. $\mathrm{M}_{22}$ & 7 & $i 1$ & ० \\
\hline 144 & 4. $\mathrm{M}_{22}$ & $0,3,11$ & $i 1, r 7$ & ○ \\
\hline 144 & 12. $\mathrm{M}_{22}$ & 7 & $z 12$ & o \\
\hline 144 & 12. $\mathrm{M}_{22}$ & $0,5,11$ & $z 12, b 7$ & ० \\
\hline 145 & $\mathrm{~S}_{4}(17)$ & $\neq 2,17$ & $b 17$ & + \\
\hline 147 & $\mathrm{O}_{8}^{+}(2)$ & 3 & & + \\
\hline 150 & $3_{1} \cdot \mathrm{U}_{4}(3)$ & 2 & $z 3, b 7$ & ○ \\
\hline 150 & $\mathrm{~S}_{4}(7)$ & $\neq 2,7$ & $b 7$ & ० \\
\hline 151 & $\mathfrak{A}_{19}$ & 3 & & + \\
\hline 151 & $\mathfrak{A}_{20}$ & 3 & & + \\
\hline
\end{tabular}


Corrigenda: Low-dimensional representations of quasi-simple groups

Table 2: Absolutely irreducible representations of quasi-simple groups, corrected

Continued from the previous page

\begin{tabular}{|c|c|c|c|c|}
\hline$d$ & $G$ & $\ell$ & field & ind \\
\hline 152 & $2 . \mathfrak{A}_{10}$ & 7 & & + \\
\hline 152 & $\mathfrak{A}_{19}$ & $\neq 3,17$ & & + \\
\hline 152 & $\mathfrak{A}_{20}$ & 2 & & + \\
\hline 152 & $\mathrm{~L}_{3}(7)$ & $\neq 3,7$ & & + \\
\hline 152 & $2 . \mathrm{O}_{8}^{+}(2)$ & 7 & & + \\
\hline 153 & $\mathfrak{A}_{12}$ & 5 & & + \\
\hline 153 & $\mathfrak{A}_{19}$ & $\neq 2,19$ & & + \\
\hline 153 & $\mathfrak{A}_{20}$ & 5 & & + \\
\hline 153 & $3_{2} \cdot \mathrm{U}_{4}(3)$ & 5 & $z 3$ & ० \\
\hline 153 & $\mathrm{~S}_{4}(4)$ & $\neq 2$ & & + \\
\hline 153 & $\mathrm{O}_{10}^{-}(2)$ & 3,5 & & + \\
\hline 153 & 3. $\mathrm{M}_{22}$ & 5 & $z 3$ & ○ \\
\hline 153 & $\mathrm{~J}_{3}$ & 3 & $b 5$ & + \\
\hline 153 & $3 . \mathrm{J}_{3}$ & 5 & $z 3$ & ० \\
\hline 153 & $3 . \mathrm{J}_{3}$ & $\neq 3,5$ & $z 3, b 5$ & ○ \\
\hline 153 & 3.McL & 5 & $z 3, b 7$ & o \\
\hline 153 & $\mathrm{He}$ & 7 & & + \\
\hline 153 & $\mathrm{He}$ & $\neq 2,7$ & $b 7$ & $\circ$ \\
\hline 153 & $3 . \mathrm{O}^{\prime} \mathrm{N}$ & 2 & $z 3$ & ० \\
\hline 154 & $\mathfrak{A}_{12}$ & $0,7,11$ & & + \\
\hline 154 & $\mathrm{~L}_{4}(5)$ & $2,3,13$ & & + \\
\hline 154 & $\mathrm{O}_{8}^{-}(2)$ & 3 & & + \\
\hline 154 & $\mathrm{O}_{10}^{-}(2)$ & $\neq 2,3,5$ & & + \\
\hline 154 & $\mathrm{M}_{22}$ & $0,7,11$ & & + \\
\hline 154 & 2. $\mathrm{M}_{22}$ & $\neq 2,5$ & $i 1$ & o \\
\hline 154 & HS & $\neq 2,5$ & & + \\
\hline 154 & $\mathrm{O}^{\prime} \mathrm{N}$ & 3 & $r 7$ & + \\
\hline 155 & $\mathfrak{A}_{10}$ & 5 & & + \\
\hline 155 & $\mathfrak{A}_{11}$ & 7 & & + \\
\hline 155 & $\mathrm{~L}_{3}(5)$ & 0,31 & & + \\
\hline 155 & $\mathrm{~L}_{3}(5)$ & 0,31 & $i 1$ & o \\
\hline 155 & $\mathrm{~L}_{4}(5)$ & 0,31 & & + \\
\hline 155 & $\mathrm{~L}_{5}(2)$ & $\neq 2$ & & + \\
\hline 155 & $\mathrm{~S}_{10}(2)$ & $\neq 2$ & & + \\
\hline 155 & $\mathrm{O}_{10}^{+}(2)$ & $\neq 2$ & & + \\
\hline 156 & 2. $\mathrm{L}_{4}(5)$ & $\neq 2,5$ & & + \\
\hline 156 & 4. $\mathrm{L}_{4}(5)$ & $\neq 2,5$ & $i 1$ & $\circ$ \\
\hline 156 & $\mathrm{U}_{3}(13)$ & $\neq 13$ & & - \\
\hline 156 & $\mathrm{~S}_{4}(5)$ & $\neq 2,5$ & & + \\
\hline 156 & $2 . \mathrm{S}_{4}(5)$ & $\neq 2,5$ & & - \\
\hline
\end{tabular}


Corrigenda: Low-dimensional representations of quasi-simple groups

Table 2: Absolutely irreducible representations of quasi-simple groups, corrected

Continued from the previous page

\begin{tabular}{|c|c|c|c|c|}
\hline$d$ & $G$ & $\ell$ & field & ind \\
\hline 157 & $\mathrm{U}_{3}(13)$ & $\neq 2,13$ & & + \\
\hline 157 & $\mathrm{U}_{3}(13)$ & $\neq 7,13$ & $z 7$ & ० \\
\hline 160 & $\mathfrak{A}_{9}$ & 2 & & + \\
\hline 160 & 2. $\mathfrak{A}_{9}$ & 0,5 & & + \\
\hline 160 & $\mathfrak{A}_{10}$ & $\neq 3,7$ & & + \\
\hline 160 & $2 . \mathfrak{A}_{10}$ & 5 & $r 6, r 21$ & + \\
\hline 160 & $2 . \mathfrak{A}_{12}$ & $0,5,7$ & $b 11$ & $\circ$ \\
\hline 160 & $2 . \mathrm{O}_{8}^{+}(2)$ & 0,5 & & + \\
\hline 160 & 2. $\mathrm{M}_{12}$ & 0,5 & $b 11$ & $\circ$ \\
\hline 160 & 4. $\mathrm{M}_{22}$ & 11 & $i 1$ & ○ \\
\hline 160 & 4. $\mathrm{M}_{22}$ & $\neq 2,11$ & $i 1, r 11$ & ० \\
\hline 160 & $\mathbf{J}_{2}$ & 0,2 & & + \\
\hline 162 & $\mathfrak{A}_{9}$ & 0,3 & & + \\
\hline 162 & $3 . G_{2}(3)$ & 2 & $z 3, b 13$ & $\circ$ \\
\hline 164 & $\mathfrak{A}_{11}$ & 2 & & - \\
\hline 164 & $\mathfrak{A}_{12}$ & 2 & & - \\
\hline 165 & $\mathfrak{A}_{11}$ & 0,11 & & + \\
\hline 165 & $\mathfrak{A}_{12}$ & $\neq 2,3$ & & + \\
\hline 165 & $\mathfrak{A}_{13}$ & 13 & & + \\
\hline 165 & $\mathrm{U}_{5}(2)$ & $\neq 2,3$ & & + \\
\hline 167 & $\mathrm{~S}_{6}(3)$ & 13 & & + \\
\hline 167 & $\mathrm{O}_{7}(3)$ & 13 & & + \\
\hline 167 & $G_{2}(3)$ & 13 & & + \\
\hline 168 & $\mathfrak{A}_{9}$ & 0,7 & & + \\
\hline 168 & 2.: $\mathfrak{A}_{9}$ & 5 & & + \\
\hline 168 & 2. $\mathfrak{A}_{9}$ & 0,7 & $i 15$ & $\circ$ \\
\hline 168 & $2 . \mathfrak{A}_{10}$ & 5 & $r 21$ & + \\
\hline 168 & $\mathrm{~S}_{6}(2)$ & $\neq 2,3$ & & + \\
\hline 168 & $2 . S_{6}(2)$ & $\neq 2,3$ & & + \\
\hline 168 & $\mathrm{~S}_{6}(3)$ & $\neq 3,13$ & & + \\
\hline 168 & $\mathrm{O}_{7}(3)$ & $0,5,7$ & & + \\
\hline 168 & $2 . \mathrm{O}_{8}^{+}(2)$ & 5 & & + \\
\hline 168 & $G_{2}(3)$ & 0,7 & & + \\
\hline 169 & $\mathfrak{A}_{20}$ & 19 & & + \\
\hline 169 & $\mathfrak{A}_{21}$ & 19 & & + \\
\hline 170 & $\mathfrak{A}_{20}$ & $\neq 2,3,19$ & & + \\
\hline 170 & $\mathrm{U}_{9}(2)$ & $\neq 2$ & & - \\
\hline 171 & $\mathfrak{A}_{20}$ & $\neq 2,5$ & & + \\
\hline 171 & $\mathfrak{A}_{21}$ & 3,7 & & + \\
\hline 171 & $3 . \mathrm{U}_{9}(2)$ & $\neq 2,3$ & $z 3$ & $\circ$ \\
\hline
\end{tabular}


Corrigenda: Low-dimensional representations of quasi-simple groups

Table 2: Absolutely irreducible representations of quasi-simple groups, corrected

Continued from the previous page

\begin{tabular}{|c|c|c|c|c|}
\hline$d$ & $G$ & $\ell$ & field & ind \\
\hline 171 & $\mathrm{~S}_{6}(7)$ & $\neq 7$ & $b 7$ & $\circ$ \\
\hline 171 & $\mathrm{O}_{8}^{-}(2)$ & 7 & & + \\
\hline 171 & $3 . J_{3}$ & $\neq 2,3$ & $z 3$ & o \\
\hline 171 & $3 . \mathrm{J}_{3}$ & $0,17,19$ & $z 3, b 5$ & ० \\
\hline 172 & $2 . \mathrm{S}_{6}(7)$ & $\neq 2,7$ & $b 7$ & ० \\
\hline 174 & $\mathrm{~S}_{4}(7)$ & 2 & & + \\
\hline 174 & 6. $\mathrm{M}_{22}$ & 11 & $z 12$ & ○ \\
\hline 174 & HS & 11 & & + \\
\hline 175 & $\mathrm{~S}_{4}(7)$ & $\neq 2,7$ & & + \\
\hline 175 & $\mathrm{O}_{8}^{+}(2)$ & $\neq 2,3$ & & + \\
\hline 175 & $\mathrm{~J}_{2}$ & $\neq 2,3$ & & + \\
\hline 175 & HS & $0,5,7$ & & + \\
\hline 176 & $\mathrm{U}_{5}(2)$ & $\neq 2,3$ & & + \\
\hline 176 & 2. $\mathrm{U}_{6}(2)$ & $\neq 2,3$ & & + \\
\hline 176 & $\mathrm{M}_{12}$ & 0,11 & & + \\
\hline 176 & 4. $\mathrm{M}_{22}$ & 0,11 & $i 1$ & $\circ$ \\
\hline 176 & 2.HS & $\neq 2,5$ & $i 1$ & ० \\
\hline 176 & 2. $\mathrm{Fi}_{22}$ & 3 & $b 13$ & + \\
\hline 180 & $\mathrm{~S}_{4}(19)$ & 2 & $b 19$ & ० \\
\hline 180 & $2 . \mathrm{S}_{4}(19)$ & $\neq 2,19$ & $b 19$ & ० \\
\hline 181 & $\mathrm{~L}_{3}(13)$ & 3,61 & & + \\
\hline 181 & $\mathrm{~S}_{4}(19)$ & $\neq 2,19$ & $b 19$ & o \\
\hline 182 & $\mathrm{~L}_{3}(13)$ & $0,2,7$ & & + \\
\hline 182 & $\mathrm{U}_{6}(3)$ & $\neq 3$ & & - \\
\hline 182 & 2. $\mathrm{U}_{6}(3)$ & $\neq 2,3$ & $i 1$ & o \\
\hline 182 & $2 . S_{6}(3)$ & $\neq 2,3$ & & - \\
\hline 182 & $2 . S_{6}(3)$ & $\neq 2,3$ & $z 3$ & $\circ$ \\
\hline 182 & $\mathrm{O}_{7}(3)$ & $\neq 2,3$ & & + \\
\hline 182 & $G_{2}(3)$ & $\neq 2,3$ & & + \\
\hline 183 & $\mathrm{~L}_{3}(13)$ & $\neq 2,13$ & & + \\
\hline 183 & $\mathrm{~L}_{3}(13)$ & $\neq 2,13$ & $i 1$ & $\circ$ \\
\hline 183 & 3. $\mathrm{L}_{3}(13)$ & $0,7,61$ & $z 12$ & ○ \\
\hline 183 & 3. $\mathrm{L}_{3}(13)$ & $\neq 3,13$ & $z 3$ & $\circ$ \\
\hline 183 & $\mathrm{U}_{6}(3)$ & $\neq 2,3$ & & + \\
\hline 185 & $\mathrm{O}_{10}^{+}(2)$ & 3,17 & & + \\
\hline 186 & $\mathfrak{A}_{11}$ & 2 & & + \\
\hline 186 & $\mathrm{~L}_{3}(5)$ & $\neq 2,5$ & & + \\
\hline 186 & $\mathrm{~S}_{10}(2)$ & 3 & & + \\
\hline 186 & $\mathrm{O}_{10}^{+}(2)$ & $\neq 2,3,17$ & & + \\
\hline 186 & $\mathrm{O}_{10}^{-}(2)$ & 3 & & + \\
\hline
\end{tabular}


Corrigenda: Low-dimensional representations of quasi-simple groups

Table 2: Absolutely irreducible representations of quasi-simple groups, corrected

Continued from the previous page

\begin{tabular}{|c|c|c|c|c|}
\hline$d$ & $G$ & $\ell$ & field & ind \\
\hline 187 & $\mathrm{~S}_{10}(2)$ & $\neq 2,3$ & & + \\
\hline 187 & $\mathrm{O}_{10}^{-}(2)$ & $\neq 2,3$ & & + \\
\hline 188 & $\mathfrak{A}_{11}$ & 5 & & + \\
\hline 188 & $\mathfrak{A}_{21}$ & 2,5 & & + \\
\hline 188 & $\mathfrak{A}_{22}$ & 2,5 & & + \\
\hline 188 & $\mathrm{U}_{4}(3)$ & 5 & & + \\
\hline 189 & $\mathfrak{A}_{9}$ & $\neq 2,5$ & & + \\
\hline 189 & $\mathfrak{A}_{21}$ & $\neq 2,5,19$ & & + \\
\hline 189 & $\mathrm{~L}_{4}(4)$ & 5 & & + \\
\hline 189 & $\mathrm{~L}_{4}(4)$ & $\neq 2,5$ & $b 5$ & + \\
\hline 189 & $3 . \mathrm{U}_{3}(8)$ & $\neq 2,3$ & $z 3$ & $\circ$ \\
\hline 189 & $\mathrm{U}_{4}(3)$ & 0,7 & & + \\
\hline 189 & $3_{2} \cdot \mathrm{U}_{4}(3)$ & $\neq 3,5$ & $z 3$ & $\circ$ \\
\hline 189 & $\mathrm{~S}_{6}(2)$ & $\neq 2,5$ & & + \\
\hline 189 & $3 . G_{2}(3)$ & 13 & $z 3$ & o \\
\hline 189 & $3 . G_{2}(3)$ & 0,7 & $z 3, b 13$ & ○ \\
\hline 189 & $\mathrm{~J}_{2}$ & 5 & & + \\
\hline 189 & $\mathbf{J}_{2}$ & $\neq 2,5$ & $b 5$ & + \\
\hline 190 & $\mathfrak{A}_{21}$ & $\neq 2,3,7$ & & + \\
\hline 190 & $\mathfrak{A}_{22}$ & 11 & & + \\
\hline 190 & $\mathrm{M}_{22}$ & 11 & & + \\
\hline 190 & $2 . \mathrm{J}_{2}$ & 5 & & - \\
\hline 194 & $\mathrm{~S}_{6}(3)$ & 7 & & + \\
\hline 194 & $\mathrm{O}_{7}(3)$ & 7 & & + \\
\hline 195 & $\mathrm{~S}_{6}(3)$ & $0,5,13$ & & + \\
\hline 195 & $\mathrm{O}_{7}(3)$ & $0,5,13$ & & + \\
\hline 196 & $\mathfrak{A}_{13}$ & 5 & & + \\
\hline 196 & $\mathfrak{A}_{14}$ & 5 & & + \\
\hline 196 & $\mathrm{~S}_{4}(8)$ & $\neq 2$ & & + \\
\hline 196 & $\mathrm{~S}_{6}(2)$ & 3 & & + \\
\hline 196 & ${ }^{3} D_{4}(2)$ & $\neq 2$ & & + \\
\hline 198 & $\mathfrak{A}_{10}$ & 2 & & + \\
\hline 198 & $\mathfrak{A}_{11}$ & 2 & & + \\
\hline 199 & $\mathfrak{A}_{10}$ & 7 & & + \\
\hline 199 & $\mathfrak{A}_{11}$ & 7 & & + \\
\hline 199 & $\mathbf{J}_{2}$ & 7 & & + \\
\hline 200 & $\mathfrak{A}_{10}$ & 2 & & + \\
\hline 200 & $2 . \mathrm{S}_{4}(7)$ & $\neq 2,7$ & $b 7$ & $\circ$ \\
\hline 201 & $\mathrm{~S}_{6}(2)$ & 7 & & + \\
\hline 202 & $2 . J_{2}$ & 5 & & - \\
\hline
\end{tabular}


Corrigenda: Low-dimensional representations of quasi-simple groups

Table 2: Absolutely irreducible representations of quasi-simple groups, corrected

Continued from the previous page

\begin{tabular}{|c|c|c|c|c|}
\hline$d$ & $G$ & $\ell$ & field & ind \\
\hline 203 & $\mathrm{~S}_{8}(2)$ & 3 & & + \\
\hline 203 & $\mathrm{O}_{8}^{-}(2)$ & 3,5 & & + \\
\hline 204 & $3_{1} \cdot \mathrm{U}_{4}(3)$ & 2 & $z 3$ & $\circ$ \\
\hline 204 & $\mathrm{U}_{5}(4)$ & $\neq 2$ & & $\circ$ \\
\hline 204 & $\mathrm{~S}_{4}(4)$ & $\neq 2,5$ & $b 5$ & + \\
\hline 204 & $\mathrm{O}_{8}^{-}(2)$ & $\neq 2,3$ & & + \\
\hline 205 & 5.U $\mathrm{U}_{5}(4)$ & $\neq 5$ & $z 5$ & ० \\
\hline 207 & $\mathfrak{A}_{13}$ & 11 & & + \\
\hline 207 & $\mathrm{~S}_{4}(4)$ & 17 & & + \\
\hline 208 & $\mathfrak{A}_{13}$ & $\neq 3,5,11$ & & + \\
\hline 208 & $\mathfrak{A}_{14}$ & 2 & & + \\
\hline 208 & $\mathfrak{A}_{22}$ & 3,7 & & + \\
\hline 208 & $\mathfrak{A}_{23}$ & 3,7 & & + \\
\hline 208 & $\mathrm{~L}_{4}(3)$ & 2,5 & & + \\
\hline 208 & 2. $\mathrm{L}_{4}(3)$ & $\neq 2,3$ & $i 2$ & ○ \\
\hline 208 & $\mathrm{~S}_{4}(5)$ & $\neq 5$ & $b 5$ & + \\
\hline 208 & $2 . S_{4}(5)$ & $\neq 2,5$ & $b 5$ & - \\
\hline 208 & $\mathrm{M}_{23}$ & 7 & & + \\
\hline 208 & 2.Suz & 3 & & - \\
\hline 209 & $\mathfrak{A}_{22}$ & $\neq 2,3,5,7$ & & + \\
\hline 209 & $\mathrm{~J}_{1}$ & $0,11,19$ & & + \\
\hline 210 & $\mathfrak{A}_{10}$ & 0,7 & & + \\
\hline 210 & $\mathfrak{A}_{11}$ & $\neq 2,11$ & & + \\
\hline 210 & $\mathfrak{A}_{12}$ & 3 & & + \\
\hline 210 & $\mathfrak{A}_{22}$ & $\neq 2,11$ & & + \\
\hline 210 & $\mathfrak{A}_{23}$ & 23 & & + \\
\hline 210 & $\mathrm{U}_{4}(3)$ & $\neq 2,3$ & & + \\
\hline 210 & 2. $\mathrm{U}_{4}(3)$ & $\neq 2,3$ & $i 1$ & ० \\
\hline 210 & $3{ }_{1} \cdot \mathrm{U}_{4}(3)$ & $\neq 2,3$ & $z 3$ & $\circ$ \\
\hline 210 & $6_{1} \cdot U_{4}(3)$ & $\neq 2,3$ & $z 3$ & $\circ$ \\
\hline 210 & $\mathrm{U}_{6}(2)$ & 3 & & + \\
\hline 210 & $3 . \mathrm{U}_{6}(2)$ & $\neq 2,3$ & $z 3$ & $\circ$ \\
\hline 210 & $\mathrm{~S}_{6}(2)$ & $\neq 2,3$ & & + \\
\hline 210 & $\mathrm{O}_{8}^{+}(2)$ & $\neq 2,3$ & & + \\
\hline 210 & $\mathrm{M}_{22}$ & $\neq 2,11$ & & + \\
\hline 210 & 2. $\mathrm{M}_{22}$ & $\neq 2,11$ & & + \\
\hline 210 & 3. $\mathrm{M}_{22}$ & $\neq 2,3$ & $z 3$ & $\circ$ \\
\hline 210 & 6. $\mathrm{M}_{22}$ & $0,5,7$ & $z 3$ & $\circ$ \\
\hline 210 & $6 . \mathrm{M}_{22}$ & $\neq 2,3$ & $z 12$ & $\circ$ \\
\hline 210 & $\mathrm{M}_{23}$ & 23 & & + \\
\hline
\end{tabular}


Corrigenda: Low-dimensional representations of quasi-simple groups

Table 2: Absolutely irreducible representations of quasi-simple groups, corrected

Continued from the previous page

\begin{tabular}{|c|c|c|c|c|}
\hline$d$ & $G$ & $\ell$ & field & ind \\
\hline 210 & HS & 5 & & + \\
\hline 210 & McL & 3,5 & & + \\
\hline 214 & $\mathbf{J}_{3}$ & 19 & $b 17, y 9$ & + \\
\hline 216 & $\mathfrak{A}_{9}$ & 0 & & + \\
\hline 216 & $2 . \mathfrak{A}_{10}$ & 0,3 & & + \\
\hline 216 & $12_{1} \cdot \mathrm{U}_{4}(3)$ & 0,7 & $z 12$ & $\circ$ \\
\hline 216 & $12_{2} \cdot U_{4}(3)$ & $\neq 2,3$ & $z 12$ & ○ \\
\hline 216 & $\mathrm{~S}_{6}(2)$ & 0 & & + \\
\hline 216 & $2 . \mathrm{J}_{2}$ & 0,3 & & - \\
\hline 217 & $\mathfrak{A}_{10}$ & 5 & & + \\
\hline 217 & $\mathrm{~L}_{5}(2)$ & $\neq 2$ & & + \\
\hline 217 & $\mathrm{~L}_{6}(2)$ & $\neq 2$ & & + \\
\hline 218 & ${ }^{3} D_{4}(3)$ & 2 & & + \\
\hline 218 & ${ }^{3} D_{4}(3)$ & 73 & & + \\
\hline 219 & ${ }^{3} D_{4}(3)$ & $\neq 2,3,73$ & & + \\
\hline 220 & $\mathfrak{A}_{13}$ & $\neq 2,13$ & & + \\
\hline 220 & $\mathfrak{A}_{14}$ & 7 & & + \\
\hline 220 & $\mathrm{U}_{4}(4)$ & 5 & & + \\
\hline 220 & $\mathrm{U}_{5}(2)$ & $\neq 2,3$ & $z 3$ & $\circ$ \\
\hline 220 & $\mathrm{M}_{23}$ & 2 & $b 7, b 23$ & ० \\
\hline 220 & $\mathrm{M}_{24}$ & 2 & $b 7, b 23$ & $\circ$ \\
\hline 220 & 2.Suz & $\neq 2,3$ & & - \\
\hline 221 & $\mathfrak{A}_{12}$ & 7 & & + \\
\hline 221 & $\mathfrak{A}_{13}$ & 7 & & + \\
\hline 221 & $\mathrm{U}_{4}(4)$ & $\neq 2$ & $b 5$ & + \\
\hline 223 & $\mathrm{~S}_{4}(7)$ & 5 & & + \\
\hline 224 & 2.: $\mathfrak{A}_{9}$ & 0,7 & & + \\
\hline 224 & $\mathfrak{A}_{10}$ & $\neq 2,5$ & & + \\
\hline 224 & 4. $U_{4}(3)$ & $\neq 2,3$ & $z 12$ & $\circ$ \\
\hline 224 & $\mathrm{~S}_{4}(7)$ & $\neq 2,5,7$ & & + \\
\hline 224 & $2 . \mathrm{O}_{8}^{+}(2)$ & $\neq 2,5$ & & + \\
\hline 224 & $\mathbf{J}_{2}$ & 0,7 & $b 5$ & + \\
\hline 225 & $\mathfrak{A}_{10}$ & 0,5 & & + \\
\hline 225 & $\mathrm{~S}_{4}(4)$ & $\neq 2,17$ & $d 17$ & + \\
\hline 225 & $\mathrm{~J}_{2}$ & $\neq 2,7$ & & + \\
\hline 229 & $\mathfrak{A}_{23}$ & 11 & & + \\
\hline 229 & $\mathfrak{A}_{24}$ & 11 & & + \\
\hline 229 & $\mathrm{U}_{6}(2)$ & 3 & & + \\
\hline 229 & $\mathrm{M}_{23}$ & 11 & & + \\
\hline 229 & $\mathrm{M}_{24}$ & 11 & & + \\
\hline
\end{tabular}


Corrigenda: Low-dimensional representations of quasi-simple groups

Table 2: Absolutely irreducible representations of quasi-simple groups, corrected

Continued from the previous page

\begin{tabular}{|c|c|c|c|c|}
\hline$d$ & $G$ & $\ell$ & field & ind \\
\hline 230 & $\mathfrak{A}_{23}$ & $\neq 3,7,11$ & & + \\
\hline 230 & $\mathfrak{A}_{24}$ & 2 & & + \\
\hline 230 & $\mathrm{M}_{23}$ & $0,5,23$ & & + \\
\hline 230 & $\mathrm{McL}$ & 2,5 & & + \\
\hline 230 & $\mathrm{Co}_{3}$ & 2,5 & & + \\
\hline 230 & $\mathrm{Co}_{2}$ & 2 & & + \\
\hline 231 & $\mathfrak{A}_{11}$ & $0,7,11$ & & + \\
\hline 231 & $\mathfrak{A}_{23}$ & $\neq 2,23$ & & + \\
\hline 231 & $\mathfrak{A}_{24}$ & 3 & & + \\
\hline 231 & $\mathrm{U}_{6}(2)$ & $\neq 2,3$ & & + \\
\hline 231 & $3 . \mathrm{U}_{6}(2)$ & $\neq 2,3$ & $z 3$ & $\circ$ \\
\hline 231 & $\mathrm{M}_{22}$ & $\neq 2,5$ & & + \\
\hline 231 & $3 . \mathrm{M}_{22}$ & $\neq 2,3$ & $z 3$ & $\circ$ \\
\hline 231 & $\mathbf{M}_{23}$ & $\neq 2,23$ & & + \\
\hline 231 & $\mathrm{M}_{23}$ & $\neq 2,3,5$ & $i 15$ & $\circ$ \\
\hline 231 & $\mathbf{M}_{24}$ & 3,5 & & + \\
\hline 231 & $\mathrm{M}_{24}$ & $\neq 2,3,5$ & $i 15$ & $\circ$ \\
\hline 231 & HS & $\neq 2,5$ & & + \\
\hline 231 & $\mathrm{McL}$ & $0,7,11$ & & + \\
\hline 231 & $\mathrm{Co}_{3}$ & 3 & & + \\
\hline 233 & $\mathfrak{A}_{13}$ & 5 & & + \\
\hline 233 & $\mathfrak{A}_{14}$ & 5 & & + \\
\hline 234 & $\mathrm{~L}_{4}(3)$ & 0,13 & & + \\
\hline 236 & $2 . \mathrm{J}_{2}$ & 3 & & - \\
\hline 238 & $\mathrm{~S}_{8}(2)$ & $\neq 2,3$ & & + \\
\hline 240 & $\mathrm{U}_{3}(16)$ & $\neq 2$ & & - \\
\hline 241 & $\mathrm{U}_{3}(16)$ & $\neq 2,17$ & $z 17$ & $\circ$ \\
\hline 244 & $\mathbf{J}_{3}$ & 2 & $b 17$ & + \\
\hline 245 & $\mathrm{O}_{8}^{-}(3)$ & 13 & & + \\
\hline 246 & $\mathrm{O}_{8}^{-}(3)$ & $\neq 3,13$ & & + \\
\hline 246 & ${ }^{2} \stackrel{\circ}{F}_{4}(2)^{\prime}$ & 2 & & + \\
\hline 246 & $\mathrm{He}$ & 2 & $b 17$ & + \\
\hline 248 & $\mathrm{~L}_{4}(5)$ & 2 & & + \\
\hline 248 & $2 . \mathrm{L}_{4}(5)$ & $\neq 2,5$ & & + \\
\hline 248 & $\mathrm{~S}_{4}(5)$ & 2 & $b 5$ & + \\
\hline 248 & Th & all & & + \\
\hline
\end{tabular}




\section{References}

1. M. Broué and J. Michel, 'Blocs et séries de Lusztig dans un groupe réductif fini', J. Reine Angew. Math. 395 (1989) 56-67. 96

2. A. Cohen, M. Liebeck, J. Saxl and G. M. Seitz, 'The local maximal subgroups of exceptional groups of Lie type, finite and algebraic', Proc. London Math. Soc. 64 (1992) 21-48. 96

3. R. Guralnick and P. H. TieP, 'Low-dimensional representations of special linear groups in cross characteristics', Proc. London Math. Soc. 78 (1999) 116-138. 95

4. G. Hiss and G. MAlle, 'Low-dimensional representations of quasi-simple groups', LMS J. Comput. Math. 4 (2001) 22-63. 96

5. G. Hiss and G. MALLE, 'Low-dimensional representations of special unitary groups', J. Algebra 236 (2001) 745-767. 96

6. G. D. JAMES, 'Representations of the symmetric groups over the field of order 2', J. Algebra 38 (1976) 280-308. 96

7. C. JANSEN, K. LuX, R. PARKer and R. Wilson, An atlas of Brauer characters (Clarendon Press, Oxford, 1995). 95

8. A. I. Kostrikin and P. H. TieP, Orthogonal decompositions and integral lattices (de Gruyter, Berlin, 1994). 96

9. P. H. Tiep and A. E. ZALessKiI, 'Minimal characters of the finite classical groups', Comm. Algebra 24 (1996) 2093-2167. 96

Gerhard Hiss Gerhard.Hiss@Math.RWTH-Aachen.DE

Lehrstuhl D für Mathematik

RWTH Aachen

Templergraben 64

D-52062 Aachen

Germany

Gunter Malle malle@mathematik.uni-kassel.de

FB Mathematik/Informatik

Universität Kassel

Heinrich-Plett-Str. 40

D-34132 Kassel

Germany. 